\title{
Increasing Storability of Mango cv. 'Zibda' Fruits By 1-Methylcyclopropene and Gibberellic Acid Postharvest Applications
}

\author{
M.A.A. Mohamed", A.F. Abd El-Khalek ${ }^{* *}$, and A.M.R.A. \\ Abdelaziz ${ }^{* * * *}$ \\ *Fruit Handling Department, Horticulture Research Institute, \\ Agricultural Research Centre, ${ }^{* *}$ Horticulture Department, \\ Faculty of Agricultural, Tanta University and ${ }^{* * *}$ Organic \\ Agricultural, Central Lab., Agricultural Research Centre, \\ Cairo, Egypt.
}

7 he effects of postharvest applications of 1-methylcyclopropene (1-MCP) and gibberellic acid $\left(\mathrm{GA}_{3}\right)$ on fruit quality of stored mango cv. 'Zibda' at $13^{\circ} \mathrm{C}$ were studied during two successive seasons, 2014 and 2015. Fruits divided into three equal groups and treated with 0,1 and $1.5 \mathrm{ppm}$ of 1-MCP. Applications were performed by placing each group into a hermetic chamber and exposing them to the gas for 24 hours at room temperature. After the duration of 1-MCP applications, the fruits in each group divided into three equal subgroups and mangoes were dipped into aqueous solutions of $\mathrm{GA}_{3}$ at 0,50 and $100 \mathrm{ppm}$ concentration for ten minutes. All treatments were stored at $13^{\circ} \mathrm{C}$ for five weeks. All postharvest applications significantly increased fruit storability and reduced the deterioration rate of mango fruits during storage. Moreover, the combination treatments of 1-MCP with $\mathrm{GA}_{3}$ were more effective in this aspect. This experiment revealed that, postharvest application of 'Zibda' mango fruits with exposed to 1-MCP for 24 hours or dipped in $\mathrm{GA}_{3}$ solution for ten minutes especially combined treatments before storage at $13^{\circ} \mathrm{C}$ alleviated decay incidence reduced weight loss percentage and increased marketable fruit percentage. In addition, these applications delayed the variability of fruit colour, decreased loss of fruit firmness and maintained inner fruit quality for up to five weeks at $13^{\circ} \mathrm{C}$ followed by three days shelf life at room temperature.

Keywords: Gibberellic acid, 1-Methylcyclopropene, Mango, Colour Firmness, Decay, Fruit quality.

Mango (Mangifera indica L.) is one of the most popular and traditional fruit in the world. In Egypt, The total occupied area of mango orchards reached 183341 feddans with annually total production of 786528 tons according to Statistics of Ministry of Agriculture and Land Reclamation (2014). Mango is considering a climacteric fruit and it has poor storage life. Fruit ripening rapidly after harvesting, this is because of trigger ethylene, which greatly affects a loss in its commercial value (Wongkhot et al., 2012). The rapid quality loss and short storage life of the mango fruit makes it highly perishable with a short shelf life period (Narayana et al., 1996). Ripening process of mango fruit is follow by a peak in respiration and a concomitant production of ethylene (Wills et al., 1998). 
Ethylene is a plant hormone, has a major role in promote ripening processes (Wang et al., 2002). This simple hydrocarbon molecule can diffuse in and out of plant tissues from both endogenous and exogenous sources (Saltveit, 1999). Ethylene affects on the quality of harvested products and can be helpful or harmful, depending on the produce and it is ripening stage (Saltveit, 1999). Usually after harvesting, the ripening process in mature green mango involves a series of biochemical reactions because of increase respiration, ethylene production and change in structural of polysaccharides. These effects resulted in fruit softening, degradation of chlorophyll, developing pigments by carotenoids biosynthesis, conversion carbohydrates or starch into sugars and change in organic acids, lipids, phenolics and volatile compounds, thus leading to fruit ripening (Herianus et al., 2003). Some commercial strategies used to withdraw deleterious effects of ethylene over fruits are practicing. For instance, avoid exposure to ethylene, minimize ethylene production and action during fruit ripening, harvest, storage and transport by using compounds that inhibit ethylene action through interaction with ethylene receptors (Sisler and Serek, 1997; Watkins, 2006 and Wang et al., 2009). Moreover, low temperature is useful for maintaining fruit quality and extending shelf life of mangoes (Mitra \& Baldwin, 1997 and Nunes et al., 2007) and it can be used to delay the ripening process of climacteric fruits by reducing the ethylene production from tissues and minimizes the rate of response of the tissue to ethylene action (Wills et al., 1998).

1-Methylcyclopropene (1-MCP) is a synthetic cyclic olefin capable of inhibiting ethylene action and it acts as a competitor of ethylene, blocking its access to the ethylene-binding receptors (Sisler and Serek, 1997). 1-MCP is a gaseous nontoxic product that is used to delay the ripening and softening process, extend the storage as well as shelf life and improves post-storage quality of several climacteric fruits (Watkins, 2006). Application of 1-MCP at low concentrations prior to the climacteric increase delayed the onset of the climacteric peaks of $\mathrm{CO}_{2}$ and ethylene production and is more effective in reduction the activities of ethylene biosynthesis enzymes, 1-aminocyclopropane1-carboxylic acid synthase (ACS), 1-aminocyclopropane-1-carboxylic acid oxidase (ACO) and 1-aminocyclopropane-1-carboxylic acid (ACC) content (Watkins, 2006 and Wang et al., 2009). Furthermore, 1-MCP was effective in inhibition the increment of the activities fruit softening enzymes (pectin methyl esterase, 1,4- $\beta$-D-glucanase and polygalacturonase) in the skin as well as in pulp tissues of 'Chousa', 'Dashehari' and 'Langra' mango fruits and consequently fruit softening delayed (Singh \& Neelam, 2008). Meanwhile, 1-MCP treatment at very low concentration has been used to delay fruit ripening, improve postharvest quality and maintain firmness of many fruits including, mangoes (Del Monte et al., 2004; Wang et al., 2009 and Sivakumar et al., 2012), avocados (Jeong et al., 2003 and Meyer \& Terry, 2010), banana (Abdalnoor, 2010), kiwifruits (Boquete et al., 2004), nectarines (Özkaya et al., 2016) and 'Eureka' lemons (Abd Elkhalek, 2012 and El-Abbasy et al., 2013). 1-MCP application is effective in preventing superficial scald and controlled soft scald, internal browning, and

Egypt. J. Hort. Vol. 43, No.2 (2016) 
breakdown incidence, impairs fruit ripening and improving overall fruit quality of pears (Vanoli et al., 2013 and Rizzolo et al., 2014). Exogenous application with $1.0 \mu \mathrm{l} / 1$ of 1 -MCP decreased ethylene production and activities of polygalacturonase and pectin methyl esterase during ripening of 'Chousa', 'Dashehari' and 'Langra' mango fruits and consequently fruit softening delayed (Singh \& Neelam, 2008). In addition, respiration rates and ethylene production are reduced in fruits treated with 1-MCP (Del Monte et al., 2004, Singh \& Neelam, 2008 and Wang et al., 2009). As for bioactive compounds, ascorbic acid content was higher in 1-MCP treated mango 'Tommy Atkins' as compared to the untreated mango (Del Monte et al., 2004). Meanwhile, 1-MCP prevented or delayed chlorophyll degradation and the activity of chlorophyllase enzyme in orange and lime fruits (Porat et al., 1999 and Win et al., 2006). Moreover, treated 'Tower II' avocado fruits with $0.9 \mu \mathrm{L} / \mathrm{L}$ of $1-\mathrm{MCP}$ for 12 hours at $20^{\circ} \mathrm{C}$ had greener colour as compared to untreated fruits after storage for 12 days at $20^{\circ} \mathrm{C}$ (Jeong et al., 2003). In addition, application of 'Tahiti' lime and 'Oroblanco' citrus fruits with 0.5 or $1.0 \mu \mathrm{L} / \mathrm{L}$ of $1-\mathrm{MCP}$ or in combination with gibberellic acid increased the shelf life for 40 days and delayed changes in colour intensity (Tavares et al., 2004). In addition, 1-MCP enhanced the disease resistance and reduced the decay incidence during storage in oranges (Porat et al., 1999), pineapples (Pietro et al., 2009), 'Eureka' lemons (Abd El-Khalek, 2012 and El-Abbasy et al., 2013) and mangoes (Chutichudet et al., 2016).

Plant growth regulators can be used as postharvest treatments to maintaining various physical and biochemical characteristics of fruits. Plant growth regulators performed effectively in reduction of postharvest decay, and extension of shelf life of mango (Ahmed \& Singh, 2000 and Feng et al., 2016). Generally, gibberellic acid $\left(\mathrm{GA}_{3}\right)$ is one of some plant growth regulators known for its anti senescing properties and ethylene antagonist, which delaying ripening processes and colour transformation of climacteric fruits (Wahdan et al., 2011). In mangoes, postharvest treatment with $\mathrm{GA}_{3}$ at 200 or $300 \mathrm{ppm}$ delayed ripening and changes in colour of fruit and reduced an increasing in total soluble solids content, total sugar, loss of ascorbic acid content, acidity and physiological loss in weight during storage and shelf life period (Feng et al., 2016). Moreover application of $\mathrm{GA}_{3}$ on mangoes significantly decreased fruit weight loss during storage (Wahdan et al., 2011 and Islam et al., 2013a). In addition, $\mathrm{GA}_{3}$ treatment significantly slowed the rate of rind colour change, softening and maintained internal fruit quality of 'Clementine' mandarin and 'Washington' navel orange fruits during cold storage (El-Otmani and Coggins, 1991). Postharvest dips in $\mathrm{GA}_{3}(50$ and $100 \mathrm{ppm}$ ) inhibited the decay development of stored lemon fruits for 90 days (Ben-Yehoshua et al., 1995).

Apparently, the reduction of losses and extension of postharvest life of mango will help to increase the market price in the off seasons, which play a good role in the economic development. Therefore, the current research was aimed at studying 
the influence of postharvest treatments with 1-MCP as an anti-ethylene and plant growth regulators $\left(\mathrm{GA}_{3}\right)$ on ripening, fruit quality parameters of 'Zibda' mango fruits during storage at $13^{\circ} \mathrm{C}$.

\section{Materials and Methods}

Fully mature green of mango fruits cv. 'Zibda' (Mangifera indica L.) were picked according to Mitra and Baldwin (1997) during the 2014 and 2015 seasons. They were obtained from a commercial private orchard in El-Behera Governorate, Egypt (latitude, $31^{\circ} 02^{\prime \prime} \mathrm{N}$, longitude, $30^{\circ} 28^{\prime \prime} \mathrm{E}$ ). The fruits were apparently uniform in size and free of visible symptoms of infection, plucked on the first week of august and directly transported to the postharvest laboratory of Horticulture Research Institute in Giza governorate, nearly at $175 \mathrm{Km}$ from the orchard. Fruits at the laboratory were thoroughly cleaned with tap water to remove dirt, latex and reduce fruit temperature, after that fruits were completely dried by electric fans. A total of 810 fresh fruits were selected and randomly divided into three equal groups. A powder containing $0.14 \%$ of $1-\mathrm{MCP}$ as active ingredient was released from a commercial powdered formulation (Smart Fresh ${ }^{\mathrm{TM}}$ Rohm and Hass Inc., USA) by adding distilled water, according to the manufacturer's instructions was used for the treatments. Three groups of mango fruits were treated as follows: the weight of 1-MCP powder was calculated by the area of hermetic chamber (162 liter), $50 \mathrm{ml}$ of distilled water were added to the flask containing a predetermined amount of powder and was stirred until its complete dissolution, which giving 0,1 and $1.5 \mathrm{ppm}$ of 1-MCP. For untreated fruits, the flask did not contain powder. 1-MCP application was performed by placing the each group into a hermetic chamber and exposing them to the gas for 24 hours. After the duration of treatments, mango fruits in each group were removed from the container and divided into three subgroups, after that the fruits were dipped in aqueous solutions of gibberellic acid (0,50 and $100 \mathrm{ppm})$ containing $0.05 \%(\mathrm{v} / \mathrm{v})$ Tween- 80 to improve wettability and adherence to mangoes surface for ten minutes. Then the fruits were air dried by electric fan for one hour and then packaged in cardboard boxes $(50 \times 35 \times 15 \mathrm{~cm})$. Each treatment included ten boxes, each containing nine fruits (90 fruits per treatment). Subsequently the treated fruits stored at $13 \pm 1^{\circ} \mathrm{C}$ and $85-90 \%$ relative humidity $(\mathrm{RH})$ for 5 weeks. At zero time of cold storage and after every one week intervals of cold storage period plus three days as a shelf life period at room conditions $\left(22-28^{\circ} \mathrm{C}\right.$ and $\left.60-75 \% \mathrm{RH}\right)$ for to simulate a marketing period, fruit samples were taken to measure the changes in external and internal fruit quality during cold storage period.

\section{Measurements of fruit physical and chemical characteristics}

Weight loss percentage was calculated by the following equation [(initial fruit weight - fruit weight at examination date) / (initial fruit weight) $] \times 100$.

Decayed fruit percentage was determined as follow [(number of decayed fruits at examination date) / (initial number of fruits)] $\times 100$.

Egypt. J. Hort. Vol. 43, No.2 (2016) 
Marketable fruit percentage was calculated by the following formula [(sound fruits at examination date) / (initial fruit weight) $\times 100$.

Fruit colour was measured using a Minolta CR-400 Chroma Meter (Minolta Co. Itd. Osaka, Japan). The measurements of skin and flesh colour were expressed in chromaticity values of hue angle $\left(h^{\circ}\right)$. Three readings were taken at different locations of each mango fruit during each data observation (McGuire, 1992 and Voss, 1992).

Fruit firmness of the flesh was recorded by using lfra texture analyzer Instrument. The force required to penetrate $1 \mathrm{~cm}$ inside the fruit using a needle probe diameter of $5 \mathrm{~mm}$ was measured. The machine was set with peak mode and speed of $0.3 \mathrm{~mm} / \mathrm{sec}$. Readings were recorded on three points in the equatorial region of the whole fruit and the results were expressed as the resistance force to the penetrating tester in units of pressure $\mathrm{g} / \mathrm{cm}^{2}$ (Watkins \& Harman, 1981).

Fruit juice was extracted by crushing the pulp of each fruit and fruit juice strained through a muslin cloth and used for measuring internal fruit quality as follow:

Fruit content of ascorbic acid (AsA) was determined according to method of adopting the procedure described by AOAC (2000) and was calculated as $\mathrm{mg} / 100 \mathrm{ml}$ juice.

Fruit content of total soluble solids (TSS) was determined by hand refractometer, 0-32 scale (ATAGO N-1 $\mathrm{E}$, Japan) and expressed in ${ }^{\circ}$ Brix after making the temperature correction at $20^{\circ} \mathrm{C}$ according to AOAC (2000).

Fruit content of titratable acidity (TA) was determined as per the procedure of AOAC (2000). Aliquot was taken and titrated against $0.1 \mathrm{~N} \mathrm{NaOH}$ to the phenolphthalein end point and expressed as citric acid and was calculated as $\mathrm{g} / 100 \mathrm{ml}$ of the juice.

Fruit TSS/TA ratio was calculated from the values recorded for fruit TSS and TA percentages determined.

\section{Statistical analysis}

This experiment was arranged in a completely randomized design having three replications (Steel et al., 1997) and consisting of two factors (postharvest treatments and storage periods). This experiment was analysis as factorial. Data calculated as percentage were transformed to arcsine of square root before statistical analysis and non-transformed means are shown. The effects of postharvest treatments and cold storage periods on different attributes were analyzed statistically by analysis of variance (ANOVA) using the MSTAT-C statistical package (M-STAT, 1993). Comparisons between means were done by Duncan's multiple range tests (DMRT) at probability $\leq 0.05$. 


\section{Results and Discussion}

Effect of postharvest applications of 1-methylcyclopropene (1-MCP) and gibberelic acid $\left(\mathrm{GA}_{3}\right)$ on 'Zibda' mango fruit quality during cold storage

Physical characteristics

Fruit weight loss, decay and marketable percentages

Data presented in Tables 1, 2 and 3 clearly indicated that, weight loss and decay percentages of mango fruits gradually and significantly increased with prolonging of cold storage period followed by shelf life during the two seasons in this work. On contrast, marketable fruits percentage gradually and significantly decreased with the advancement of cold storage period in the two seasons under this investigation.

TABLE 1. Effect of postharvest treatments of 1-methylcyclopropene (1-MCP) and gibberelic acid $\left(\mathrm{GA}_{3}\right)$ on changes in weight loss percentage of 'Zibda' mango fruits during storage at $13^{\circ} \mathrm{C}$ plus 3 days shelf life at $22-28^{\circ} \mathrm{C}$

\begin{tabular}{|c|c|c|c|c|c|c|c|c|c|}
\hline \multirow{2}{*}{ Postharvest treatments } & \multicolumn{7}{|c|}{ Storage period (days) } & \multirow{2}{*}{\multicolumn{2}{|c|}{ Means }} \\
\hline & $\mathbf{0}$ & 7 & 14 & 2 & & 28 & 35 & & \\
\hline \multicolumn{10}{|c|}{ Season 2014} \\
\hline Distilled water (control) & $2.77 \mathrm{r}-\mathrm{v}$ & 4.47 opq & $7.13 \mathrm{mn}$ & 10.17 & $e-j$ & $15.73 \mathrm{~b}$ & 20.87 a & 10.19 & A \\
\hline 1 ppm 1-MCP & $1.03 \mathrm{wx}$ & $2.73 \mathrm{r}-\mathrm{v}$ & 6.03 no & 8.80 & $\mathrm{jkl}$ & $11.57 \mathrm{def}$ & $12.33 \mathrm{~cd}$ & 7.08 & $\mathrm{BCD}$ \\
\hline 1.5 ppm 1-MCP & $0.47 \mathrm{x}$ & $2.17 \mathrm{r}-\mathrm{w}$ & 5.53 o & 8.90 & i-l & $11.23 \mathrm{def}$ & $12.47 \mathrm{~cd}$ & 6.79 & $\mathrm{CDE}$ \\
\hline $50 \mathrm{ppm} \mathrm{GA} 3$ & $1.27 \mathrm{vwx}$ & $2.97 \mathrm{q}-\mathrm{u}$ & 5.90 no & 9.93 & $f-j$ & $11.50 \mathrm{def}$ & $13.27 \mathrm{c}$ & 7.47 & B \\
\hline $100 \mathrm{ppm} \mathrm{GA}_{3}$ & $2.70 \mathrm{r}-\mathrm{v}$ & $4.40 \mathrm{opq}$ & $5.53 \mathrm{o}$ & 8.13 & $\mathrm{klm}$ & 11.77 cde & $11.80 \mathrm{cde}$ & 7.39 & $\mathrm{BC}$ \\
\hline $1 \mathrm{ppm} 1-\mathrm{MCP}+50 \mathrm{ppm} \mathrm{GA}$ & $1.70 \mathrm{t}-\mathrm{x}$ & $3.40 \mathrm{p}-\mathrm{s}$ & $4.50 \mathrm{opq}$ & 9.27 & h-l & $10.93 \mathrm{~d}-\mathrm{g}$ & 11.40 def & 6.87 & $\mathrm{CD}$ \\
\hline $1.5 \mathrm{ppm} 1-\mathrm{MCP}+50 \mathrm{ppm} \mathrm{GA}_{3}$ & 1.84 s-x & $3.54 \mathrm{pqr}$ & 4.69 op & 8.60 & $\mathrm{j}-\mathrm{m}$ & $11.50 \mathrm{def}$ & 11.70 cde & 6.98 & $\mathrm{BCD}$ \\
\hline $1 \mathrm{ppm} 1-\mathrm{MCP}+100 \mathrm{ppm} \mathrm{GA}$ & $1.07 \mathrm{wx}$ & $2.77 \mathrm{r}-\mathrm{v}$ & 5.70 no & 8.33 & $\mathrm{klm}$ & $10.47 \mathrm{e}-\mathrm{i}$ & $11.30 \mathrm{def}$ & 6.61 & $\mathrm{DE}$ \\
\hline $1.5 \mathrm{ppm} 1-\mathrm{MCP}+100 \mathrm{ppm} \mathrm{GA}_{3}$ & $1.53 \mathrm{u}-\mathrm{x}$ & $3.23 \mathrm{p}-\mathrm{t}$ & $4.70 \mathrm{op}$ & 7.80 & $\operatorname{lm}$ & $9.50 \mathrm{~g}-\mathrm{k}$ & $10.63 \mathrm{e}-\mathrm{h}$ & 6.23 & $\mathrm{E}$ \\
\hline Means & $1.60 \mathrm{~F}$ & $3.30 \mathrm{E}$ & $5.52 \mathrm{D}$ & 8.88 & $\mathrm{C}$ & $11.58 \mathrm{~B}$ & $12.86 \mathrm{~A}$ & & \\
\hline \multicolumn{10}{|c|}{ Season 2015} \\
\hline Distilled water (control) & $4.18 \mathrm{r}-\mathrm{v}$ & $5.88 \mathrm{n}-\mathrm{r}$ & $7.72 \mathrm{lmn}$ & 11.20 & $f-i$ & $17.47 \mathrm{~b}$ & $19.90 \mathrm{a}$ & 11.06 & $\mathrm{~A}$ \\
\hline 1 ppm 1-MCP & $1.75 \mathrm{w}-\mathrm{Z}$ & $3.45 \mathrm{t}-\mathrm{w}$ & $5.34 \mathrm{o}-\mathrm{s}$ & 7.12 & mno & $12.12 \mathrm{~d}-\mathrm{h}$ & $13.50 \mathrm{~cd}$ & 7.21 & $\mathrm{C}$ \\
\hline $1.5 \mathrm{ppm} 1-\mathrm{MCP}$ & $1.71 \mathrm{w}-\mathrm{z}$ & $3.41 \mathrm{t}-\mathrm{w}$ & $6.09 \mathrm{n}-\mathrm{q}$ & 8.35 & $\mathrm{klm}$ & $11.40 \mathrm{f}-\mathrm{i}$ & $11.52 \mathrm{f}-\mathrm{i}$ & 7.08 & $\mathrm{C}$ \\
\hline $50 \mathrm{ppm} \mathrm{GA} 3$ & $3.29 \mathrm{t}-\mathrm{x}$ & $4.99 \mathrm{p}-\mathrm{t}$ & $6.57 \mathrm{~m}-\mathrm{p}$ & 9.79 & $\mathrm{ijk}$ & $13.37 \mathrm{cde}$ & $12.46 \mathrm{c}-\mathrm{g}$ & 8.41 & B \\
\hline $100 \mathrm{ppm} \mathrm{GA} 3$ & $2.75 \mathrm{u}-\mathrm{x}$ & $4.45 \mathrm{q}-\mathrm{u}$ & $6.18 \mathrm{n}-\mathrm{q}$ & 8.95 & $\mathrm{jkl}$ & $13.01 \mathrm{c}-\mathrm{f}$ & $13.97 \mathrm{c}$ & 8.22 & B \\
\hline $1 \mathrm{ppm} 1-\mathrm{MCP}+50 \mathrm{ppm} \mathrm{GA}$ & $0.81 \mathrm{z}$ & $2.51 \mathrm{v}-\mathrm{z}$ & $3.97 \mathrm{~s}-\mathrm{v}$ & 7.57 & $\operatorname{lmn}$ & $12.74 \mathrm{c}-\mathrm{g}$ & $11.84 \mathrm{~d}-\mathrm{h}$ & 6.57 & $\mathrm{CD}$ \\
\hline 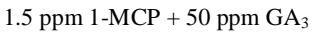 & $1.57 \mathrm{xyz}$ & $3.27 \mathrm{t}-\mathrm{x}$ & $5.10 \mathrm{p}-\mathrm{t}$ & 7.58 & $\operatorname{lmn}$ & $11.62 \mathrm{e}-\mathrm{h}$ & 10.57 hij & 6.62 & $\mathrm{CD}$ \\
\hline $1 \mathrm{ppm} 1-\mathrm{MCP}+100 \mathrm{ppm} \mathrm{GA} 3$ & $0.94 \mathrm{yz}$ & $2.64 u-y$ & $4.06 \mathrm{~s}-\mathrm{v}$ & 6.19 & $n-q$ & 10.97 ghi & $12.24 \mathrm{c}-\mathrm{h}$ & 6.17 & $\mathrm{D}$ \\
\hline $1.5 \mathrm{ppm} 1-\mathrm{MCP}+100 \mathrm{ppm} \mathrm{GA_{3 }}$ & $1.82 \mathrm{w}-\mathrm{z}$ & $3.52 \mathrm{~s}-\mathrm{w}$ & $4.80 \mathrm{p}-\mathrm{t}$ & 7.67 & $\operatorname{lmn}$ & $10.43 \mathrm{hij}$ & $9.07 \mathrm{jkl}$ & 6.22 & $\mathrm{D}$ \\
\hline Means & $2.09 \mathrm{E}$ & $3.79 \mathrm{D}$ & $5.54 \mathrm{C}$ & 8.27 & B & $12.57 \mathrm{~A}$ & $12.79 \mathrm{~A}$ & & \\
\hline
\end{tabular}

Means followed by the same letters within postharvest treatments, storage periods and their interactions in each season are not significantly different at level $P \leq 0.05$ according to DMRT.

The duration of the exposure to $1-\mathrm{MCP}$ and $\mathrm{GA}_{3}$ dipping were 24 hours and 10 minutes, respectively.

Egypt. J. Hort. Vol. 43, No.2 (2016) 
These results are in accordance with those reported by Islam et al. (2013a). They reported that, mango fruits weight loss percentage and decay incidence gradually and significantly increased with prolonging of storage period.

Data also indicated that, all postharvest treatments significantly decreased the deterioration rate in these characteristics during the two seasons in this work as compared to untreated fruits. Moreover, fruits treated with either 1 or 1.5 from 1 MCP in combination with gibberellic acid at 50 or $100 \mathrm{ppm}$ significantly had the lowest deterioration rate in these characteristics during the two seasons under this study. Data also cleared that, there was a significant interaction among all these studded treatments during the two seasons in this research.

TABLE 2. Effect of postharvest treatments of 1-methylcyclopropene (1-MCP) and gibberelic acid $\left(\mathrm{GA}_{3}\right)$ on changes in decay percentage of 'Zibda' mango fruits during storage at $13^{\circ} \mathrm{C}$ plus 3 days shelf life at $22-28^{\circ} \mathrm{C}$

\begin{tabular}{|c|c|c|c|c|c|c|c|c|c|c|}
\hline \multirow{2}{*}{ Postharvest treatments } & \multicolumn{8}{|c|}{ Storage period (days) } & \multirow{2}{*}{\multicolumn{2}{|c|}{ Means }} \\
\hline & $\mathbf{0}$ & 7 & 14 & & 2 & 1 & 28 & 35 & & \\
\hline \multicolumn{11}{|c|}{ Season 2014} \\
\hline Distilled water (control) & $0.00 \mathrm{~m}$ & $0.00 \mathrm{~m}$ & 9.73 & $\mathrm{ij}$ & 20.03 & $\mathrm{c}$ & $37.27 \mathrm{~b}$ & 41.77 a & 18.13 & A \\
\hline 1 ppm 1-MCP & $0.00 \mathrm{~m}$ & $0.00 \mathrm{~m}$ & 0.00 & $\mathrm{~m}$ & 0.00 & $\mathrm{~m}$ & $11.80 \mathrm{f}-\mathrm{i}$ & $14.07 \mathrm{~d}-\mathrm{f}$ & 4.31 & B-D \\
\hline $1.5 \mathrm{ppm} 1-\mathrm{MCP}$ & $0.00 \mathrm{~m}$ & $0.00 \mathrm{~m}$ & 0.00 & $\mathrm{~m}$ & 0.00 & $\mathrm{~m}$ & $10.67 \mathrm{~g}-\mathrm{j}$ & $12.37 \mathrm{f}-\mathrm{h}$ & 3.84 & C-E \\
\hline $50 \mathrm{ppm} \mathrm{GA} 3$ & $0.00 \mathrm{~m}$ & $0.00 \mathrm{~m}$ & 0.00 & $\mathrm{~m}$ & 0.00 & $\mathrm{~m}$ & $13.23 \mathrm{ef}$ & $15.73 \mathrm{~d}$ & 4.83 & B \\
\hline $100 \mathrm{ppm} \mathrm{GA}_{3}$ & $0.00 \mathrm{~m}$ & $0.00 \mathrm{~m}$ & 0.00 & $\mathrm{~m}$ & 0.00 & $\mathrm{~m}$ & $12.60 \mathrm{e}-\mathrm{g}$ & $14.67 \mathrm{de}$ & 4.54 & $\mathrm{BC}$ \\
\hline $1 \mathrm{ppm} \mathrm{1-MCP}+50 \mathrm{ppm} \mathrm{GA}$ & $0.00 \mathrm{~m}$ & $0.00 \mathrm{~m}$ & 0.00 & $\mathrm{~m}$ & 0.00 & $\mathrm{~m}$ & $9.57 \mathrm{j}$ & $12.20 \mathrm{f}-\mathrm{h}$ & 3.63 & $\mathrm{DE}$ \\
\hline $1.5 \mathrm{ppm} 1-\mathrm{MCP}+50 \mathrm{ppm} \mathrm{GA} 3$ & $0.00 \mathrm{~m}$ & $0.00 \mathrm{~m}$ & 0.00 & $\mathrm{~m}$ & 0.00 & $\mathrm{~m}$ & $7.29 \mathrm{k}$ & 12.90 ef & 3.36 & $\mathrm{E}$ \\
\hline $1 \mathrm{ppm} 1-\mathrm{MCP}+100 \mathrm{ppm} \mathrm{GA}$ & $0.00 \mathrm{~m}$ & $0.00 \mathrm{~m}$ & 0.00 & $\mathrm{~m}$ & 0.00 & $\mathrm{~m}$ & $0.00 \mathrm{~m}$ & $13.97 \mathrm{~d}-\mathrm{f}$ & 2.33 & $\mathrm{~F}$ \\
\hline $1.5 \mathrm{ppm} 1-\mathrm{MCP}+100 \mathrm{ppm} \mathrm{GA}_{3}$ & $0.00 \mathrm{~m}$ & $0.00 \mathrm{~m}$ & 0.00 & $\mathrm{~m}$ & 0.00 & $\mathrm{~m}$ & $3.07 \quad 1$ & $10.23 \mathrm{~h}-\mathrm{j}$ & 2.22 & $\mathrm{~F}$ \\
\hline Means & $0.00 \mathrm{E}$ & $0.00 \mathrm{E}$ & 1.08 & $\mathrm{D}$ & 2.23 & $\mathrm{C}$ & $11.72 \mathrm{~B}$ & $16.43 \mathrm{~A}$ & & \\
\hline \multicolumn{11}{|c|}{ Season 2015} \\
\hline Distilled water (control) & $0.00 \mathrm{~g}$ & $0.00 \mathrm{~g}$ & 12.28 & de & 18.49 & $\mathrm{~cd}$ & $30.17 \mathrm{~b}$ & $43.31 \mathrm{a}$ & 17.37 & A \\
\hline $1 \mathrm{ppm} 1-\mathrm{MCP}$ & $0.00 \mathrm{~g}$ & $0.00 \mathrm{~g}$ & 0.00 & $\mathrm{~g}$ & 0.00 & $\mathrm{~g}$ & $5.39 \mathrm{fg}$ & $18.59 \mathrm{~cd}$ & 4.00 & $\mathrm{BC}$ \\
\hline $1.5 \mathrm{ppm} 1-\mathrm{MCP}$ & $0.00 \mathrm{~g}$ & $0.00 \mathrm{~g}$ & 0.00 & $\mathrm{~g}$ & 0.00 & $\mathrm{~g}$ & $5.03 \mathrm{fg}$ & $17.15 \mathrm{c}-\mathrm{e}$ & 3.70 & $\mathrm{BC}$ \\
\hline $50 \mathrm{ppm} \mathrm{GA}_{3}$ & $0.00 \mathrm{~g}$ & $0.00 \mathrm{~g}$ & 0.00 & $\mathrm{~g}$ & 0.00 & $\mathrm{~g}$ & 11.23 ef & $20.79 \mathrm{c}$ & 5.34 & $\mathrm{~B}$ \\
\hline $100 \mathrm{ppm} \mathrm{GA}_{3}$ & $0.00 \mathrm{~g}$ & $0.00 \mathrm{~g}$ & 0.00 & $\mathrm{~g}$ & 0.00 & $\mathrm{~g}$ & $5.42 \mathrm{fg}$ & $20.41 \mathrm{c}$ & 4.31 & $\mathrm{BC}$ \\
\hline $1 \mathrm{ppm} \mathrm{1-MCP}+50 \mathrm{ppm} \mathrm{GA} 3$ & $0.00 \mathrm{~g}$ & $0.00 \mathrm{~g}$ & 0.00 & $\mathrm{~g}$ & 0.00 & $\mathrm{~g}$ & $0.00 \mathrm{~g}$ & $17.65 \mathrm{c}-\mathrm{e}$ & 2.94 & $\mathrm{BC}$ \\
\hline $1.5 \mathrm{ppm} 1-\mathrm{MCP}+50 \mathrm{ppm} \mathrm{GA}$ & $0.00 \mathrm{~g}$ & $0.00 \mathrm{~g}$ & 0.00 & $\mathrm{~g}$ & 0.00 & $\mathrm{~g}$ & $0.00 \mathrm{~g}$ & $17.19 \mathrm{c}-\mathrm{e}$ & 2.86 & $\mathrm{BC}$ \\
\hline $1 \mathrm{ppm} 1-\mathrm{MCP}+100 \mathrm{ppm} \mathrm{GA}_{3}$ & $0.00 \mathrm{~g}$ & $0.00 \mathrm{~g}$ & 0.00 & $\mathrm{~g}$ & 0.00 & $\mathrm{~g}$ & $0.00 \mathrm{~g}$ & $14.35 \mathrm{c}-\mathrm{e}$ & 2.39 & $\mathrm{C}$ \\
\hline $1.5 \mathrm{ppm} 1-\mathrm{MCP}+100 \mathrm{ppm} \mathrm{GA}_{3}$ & $0.00 \mathrm{~g}$ & $0.00 \mathrm{~g}$ & 0.00 & $\mathrm{~g}$ & 0.00 & $\mathrm{~g}$ & $0.00 \mathrm{~g}$ & $14.14 \mathrm{c}-\mathrm{e}$ & 2.36 & $\mathrm{C}$ \\
\hline Means & $0.00 \mathrm{C}$ & $0.00 \mathrm{C}$ & 1.36 & $\mathrm{C}$ & 2.05 & $\mathrm{C}$ & $6.36 \mathrm{~B}$ & $20.40 \mathrm{~A}$ & & \\
\hline
\end{tabular}

Means followed by the same letters within postharvest treatments, storage periods and their interactions in each season are not significantly different at level $P \leq 0.05$ according to DMRT.

The duration of the exposure to $1-\mathrm{MCP}$ and $\mathrm{GA}_{3}$ dipping were 24 hours and 10 minutes, respectively. 
Fruit weight loss during storage could largely be attributed to moisture loss that occurs through physiological processes, such as transpiration and respiration (Magazin et al., 2010). The reduction in weight loss of the fruits treated with 1MCP and $\mathrm{GA}_{3}$ especially in combination treatments might be due to its antisenescent action, which causes the decrease in the tissue permeability thereby reduced physiological loss in weight by delaying fruit ripening as mentioned by Sudha et al. (2007).

TABLE 3. Effect of postharvest treatments of 1-methylcyclopropene (1-MCP) and gibberelic acid $\left(\mathrm{GA}_{3}\right)$ on changes in marketable percentage of 'Zibda' mango fruits during storage at $13^{\circ} \mathrm{C}$ plus 3 days shelf life at $22-28^{\circ} \mathrm{C}$

\begin{tabular}{|c|c|c|c|c|c|c|c|}
\hline \multirow{2}{*}{ Postharvest treatments } & \multicolumn{6}{|c|}{ Storage period (days) } & \multirow[b]{2}{*}{ Means } \\
\hline & 0 & 7 & 14 & 21 & 28 & 35 & \\
\hline \multicolumn{8}{|c|}{ Season 2014} \\
\hline Distilled water (control) & 97.23 a-e & $95.53 \mathrm{~d}-\mathrm{h}$ & 83.13 о & $69.99 \mathrm{u}$ & $47.00 \mathrm{v}$ & $37.37 \mathrm{w}$ & $71.71 \mathrm{E}$ \\
\hline 1 ppm 1-MCP & $98.97 \mathrm{ab}$ & 97.27 a-e & $93.97 \mathrm{~h}-\mathrm{k}$ & $91.20 \mathrm{~lm}$ & $76.63 \mathrm{rs}$ & $73.60 \mathrm{t}$ & $88.61 \mathrm{CD}$ \\
\hline $1.5 \mathrm{ppm} 1-\mathrm{MCP}$ & 99.53 a & 97.83 a-e & $94.47 \mathrm{f}-\mathrm{i}$ & $91.10 \mathrm{~lm}$ & $78.10 \mathrm{qr}$ & $75.17 \mathrm{st}$ & $89.37 \mathrm{BC}$ \\
\hline $50 \mathrm{ppm} \mathrm{GA}$ & $98.73 \mathrm{ab}$ & 97.03 a-f & $94.10 \mathrm{~g}-\mathrm{k}$ & $89.83 \mathrm{~lm}$ & $75.27 \mathrm{st}$ & $71.00 \mathrm{u}$ & $87.66 \mathrm{D}$ \\
\hline $100 \mathrm{ppm} \mathrm{GA} 3$ & 97.30 a-e & $95.60 \mathrm{c}-\mathrm{h}$ & $94.47 \mathrm{f}-\mathrm{i}$ & $91.87 \mathrm{j}-\mathrm{m}$ & $75.63 \mathrm{st}$ & $73.53 \mathrm{t}$ & $88.07 \mathrm{D}$ \\
\hline $1 \mathrm{ppm} \mathrm{1-MCP}+50$ ppm GA 3 & $98.30 \mathrm{abc}$ & $96.60 \mathrm{~b}-\mathrm{h}$ & $95.50 \mathrm{~d}-\mathrm{h}$ & $90.73 \mathrm{~lm}$ & $79.50 \mathrm{pq}$ & $76.40 \mathrm{rs}$ & $89.51 \mathrm{BC}$ \\
\hline $1.5 \mathrm{ppm} 1-\mathrm{MCP}+50 \mathrm{ppm} \mathrm{GA}$ & 98.16 a-d & $96.46 \mathrm{~b}-\mathrm{h}$ & 95.31 e-h & $91.40 \mathrm{~lm}$ & 81.21 op & $75.40 \mathrm{st}$ & 89.66 B \\
\hline $1 \mathrm{ppm} 1-\mathrm{MCP}+100 \mathrm{ppm} \mathrm{GA}$ & $98.93 \mathrm{ab}$ & 97.23 a-e & $94.30 \mathrm{~g}-\mathrm{j}$ & $91.67 \mathrm{klm}$ & $89.53 \mathrm{mn}$ & $74.73 \mathrm{st}$ & $91.07 \mathrm{~A}$ \\
\hline $1.5 \mathrm{ppm} 1-\mathrm{MCP}+100 \mathrm{ppm} \mathrm{GA}_{3}$ & $98.47 \mathrm{ab}$ & $96.77 \mathrm{~b}-\mathrm{g}$ & 95.30 e-h & $92.20 \mathrm{i}-1$ & $87.43 \mathrm{n}$ & $79.14 \mathrm{pq}$ & $91.55 \mathrm{~A}$ \\
\hline Means & $98.40 \mathrm{~A}$ & $96.70 \mathrm{~B}$ & $93.39 \mathrm{C}$ & $88.89 \mathrm{D}$ & $76.70 \mathrm{E}$ & $70.70 \mathrm{~F}$ & \\
\hline \multicolumn{8}{|c|}{ Season 2015} \\
\hline Distilled water (control) & 95.82 a-e & 94.12 a-g & $80.00 \mathrm{klm}$ & 70.32 nop & $52.35 \mathrm{q}$ & $36.80 \mathrm{r}$ & $71.57 \mathrm{D}$ \\
\hline 1 ppm 1-MCP & $98.25 \mathrm{ab}$ & 96.55 a-d & 94.66 a-f & 92.88 a-g & 82.49 i-1 & 67.92 op & $88.79 \mathrm{ABC}$ \\
\hline $1.5 \mathrm{ppm} 1-\mathrm{MCP}$ & $98.29 \mathrm{ab}$ & 96.59 a-d & $93.91 \mathrm{a}-\mathrm{g}$ & $91.65 \mathrm{a}-\mathrm{g}$ & 83.57 h-k & 71.33 nop & $89.22 \mathrm{AB}$ \\
\hline $50 \mathrm{ppm} \mathrm{GA}$ & 96.71 a-d & $95.01 \mathrm{a}-\mathrm{f}$ & $93.43 \mathrm{a}-\mathrm{g}$ & $90.21 \mathrm{c}-\mathrm{h}$ & $75.39 \mathrm{mn}$ & 66.75 op & $86.25 \mathrm{C}$ \\
\hline $100 \mathrm{ppm} \mathrm{GA} 3$ & $97.25 \mathrm{abc}$ & 95.55 a-f & 93.82 a-g & $91.05 \mathrm{~b}-\mathrm{g}$ & $81.56 \mathrm{j}-\mathrm{m}$ & $65.62 \mathrm{p}$ & $87.48 \mathrm{BC}$ \\
\hline $1 \mathrm{ppm} 1-\mathrm{MCP}+50 \mathrm{ppm} \mathrm{GA} 3$ & $99.19 \mathrm{a}$ & $97.49 \mathrm{abc}$ & 96.03 a-e & $92.43 \mathrm{a}-\mathrm{g}$ & $87.26 \mathrm{~g}-\mathrm{j}$ & 70.51 nop & $90.49 \mathrm{~A}$ \\
\hline $1.5 \mathrm{ppm} 1-\mathrm{MCP}+50 \mathrm{ppm} \mathrm{\textrm {GA } _ { 3 }}$ & $98.43 \mathrm{ab}$ & 96.73 a-d & 94.90 a-f & $92.42 \mathrm{a}-\mathrm{g}$ & $88.38 \mathrm{f}-\mathrm{i}$ & 72.25 nop & $90.52 \mathrm{~A}$ \\
\hline $1 \mathrm{ppm} 1-\mathrm{MCP}+100 \mathrm{ppm} \mathrm{GA}$ & $99.06 \mathrm{a}$ & $97.36 \mathrm{abc}$ & 95.94 a-e & $93.81 \mathrm{a}-\mathrm{g}$ & $89.03 \mathrm{e}-\mathrm{i}$ & 73.41 no & $91.44 \mathrm{~A}$ \\
\hline $1.5 \mathrm{ppm} 1-\mathrm{MCP}+100 \mathrm{ppm} \mathrm{GA}_{3}$ & $98.18 \mathrm{ab}$ & 96.48 a-e & 95.20 a-f & $92.33 \mathrm{a}-\mathrm{g}$ & $89.57 \mathrm{~d}-\mathrm{h}$ & $76.79 \operatorname{lmn}$ & $91.43 \mathrm{~A}$ \\
\hline Means & $97.91 \mathrm{~A}$ & $96.21 \mathrm{~A}$ & $93.10 \mathrm{~B}$ & $89.68 \mathrm{C}$ & $81.07 \mathrm{D}$ & $66.82 \mathrm{E}$ & \\
\hline
\end{tabular}

These results are in harmony with the findings of Chutichudet et al. (2016) on mango fruits, who reported that postharvest application of 1-MCP reduced physiological loss in weight in comparison to untreated fruits. Moreover, these results are conformity with the findings of Wahdan et al. (2011) and Islam et al. (2013a). They reported that, dipping mango fruits in $\mathrm{GA}_{3}$ reduced weight loss during storage.

Egypt. J. Hort. Vol. 43, No.2 (2016) 
Susceptibility mango fruits to postharvest diseases were increased during storage because of the physiological changes and senescence of fruits (Prusky \& Keen, 1993). They also cleared that, the reducing decay appearance after treating with 1-MCP may be associated with changes in natural antifungal compounds in ripening fruit.

The reduction of decay incidence of mangoes cv. 'Zibda' during storage by postharvest application with either 1-MCP or $\mathrm{GA}_{3}$ especially in combination treatments may be due to the low respiration rate and the delay senescence of fruits, which could enhance resistance to infection and lesion development as has been previously suggested by Watkins (2008).

These results coincide with previous reports of Pietro et al. (2009) on pineapple fruits, who found that $1-\mathrm{MCP}$ might be beneficial in controlling microbial growth. Moreover, these results are in line with the findings of Abd Elkhalek (2012) and El-Abbasy et al. (2013) on 'Eureka' lemon fruits and Chutichudet et al. (2016) on mangoes. They demonstrated that, 1-MCP application had a positive effect in reducing postharvest decay as compared to control treatment during storage. Moreover, immersed fruits before storage in $\mathrm{GA}_{3}$ inhibited the decay development of mangoes (Kumar \& Sing, 1993) and lemons (Ben-Yehoshua el al., 1995).

\section{Fruit firmness}

Data shown in Table 4 appeared that, firmness of mango fruits gradually and significantly decreased with prolonging of cold storage period followed by shelf life during the two seasons in this study. These results are in harmony with those obtained by Singh \& Neelam (2008), Wang et al. (2009), Sivakumar et al. (2012) and Chutichudet et al. (2016). They illustrated that, firmness of mango fruits gradually and significantly decreased with the progress of storage period.

Moreover, data indicated that, all postharvest treatments significantly decreased loss of fruit firmness during the two seasons in this work as compared to untreated fruits. Moreover, fruits treated with 1.5 of 1-MCP in combination with gibberellic acid at $100 \mathrm{ppm}$ significantly had the lowest decreasing rate of fruit firmness during the two seasons under this study. Data also mentioned that, there was a significant interaction among all these studded treatments during the two seasons in this research.

Fruit softening of mango fruits was associated with the increase of activities of cell wall hydrolysis enzymes because of ripening process (Ali et al., 2004). Similar effects in delaying softening of mango fruits and reducing the deterioration rate of firmness during storage have been observed by 1-MCP postharvest treatments (Del Monte et al., 2004; Singh \& Neelam, 2008; Wang et al., 2009; Sivakumar et al., 2012 and Chutichudet et al., 2016). Furthermore, these results are in harmony with those obtained by Wahdan et al. (2011), who 
found that postharvest treatment of $\mathrm{GA}_{3}$ of mango fruits showed better retention of firmness as compared to untreated fruits.

TABLE 4. Effect of postharvest treatments of 1-methylcyclopropene (1-MCP) and gibberelic acid $\left(\mathrm{GA}_{3}\right)$ on changes in firmness $\left(\mathrm{g} / \mathrm{cm}^{2}\right)$ of 'Zibda' mango fruits during storage at $13^{\circ} \mathrm{C}$ plus 3 days shelf life at $22-28^{\circ} \mathrm{C}$

\begin{tabular}{|c|c|c|c|c|c|c|c|}
\hline \multirow{2}{*}{ Postharvest treatments } & \multicolumn{6}{|c|}{ Storage period (days) } & \multirow[b]{2}{*}{ Means } \\
\hline & $\mathbf{0}$ & 7 & 14 & 21 & 28 & 35 & \\
\hline \multicolumn{8}{|c|}{ Season 2014} \\
\hline Distilled water (control) & $195.7 \mathrm{de}$ & 192.0 ef & $164.3 \mathrm{ij}$ & $93.7 \mathrm{n}$ & 48.3 uv & $36.3 \mathrm{v}$ & $121.7 \mathrm{D}$ \\
\hline $1 \mathrm{ppm} 1-\mathrm{MCP}$ & 193.0 ef & $189.3 \mathrm{efg}$ & $156.3 \mathrm{j}$ & $108.7 \mathrm{~lm}$ & $98.3 \mathrm{mn}$ & $57.3 \mathrm{stu}$ & $133.8 \mathrm{C}$ \\
\hline $1.5 \mathrm{ppm} 1-\mathrm{MCP}$ & 200.4 a-e & 196.7 cde & $175.0 \mathrm{ghi}$ & $115.3 \mathrm{kl}$ & 77.3 o-r & $64.7 \mathrm{rst}$ & $138.2 \mathrm{ABC}$ \\
\hline $50 \mathrm{ppm} \mathrm{GA} 3$ & $213.4 \mathrm{ab}$ & $209.7 \mathrm{a}-\mathrm{d}$ & $174.7 \mathrm{ghi}$ & 86.0 nop & $74.7 \mathrm{pqr}$ & $55.7 \mathrm{tu}$ & $135.7 \mathrm{C}$ \\
\hline $100 \mathrm{ppm} \mathrm{GA} 3$ & $215.0 \mathrm{a}$ & $211.3 \mathrm{abc}$ & $170.3 \mathrm{hij}$ & $97.0 \mathrm{mn}$ & $70.7 \mathrm{q}-\mathrm{t}$ & $55.7 \mathrm{tu}$ & $136.7 \mathrm{BC}$ \\
\hline $1 \mathrm{ppm} 1-\mathrm{MCP}+50 \mathrm{ppm} \mathrm{GA}$ & 191.4 ef & 187.7 efg & 168.0 hij & $116.7 \mathrm{kl}$ & $92.3 \mathrm{n}$ & $68.3 \mathrm{rst}$ & 137.4 ABC \\
\hline $1.5 \mathrm{ppm} 1-\mathrm{MCP}+50 \mathrm{ppm} \mathrm{GA}$ & 202.4 a-e & 198.7 b-e & $180.0 \mathrm{fgh}$ & $127.3 \mathrm{k}$ & 83.3 n-q & $65.0 \mathrm{rst}$ & $142.8 \mathrm{~A}$ \\
\hline $1 \mathrm{ppm} 1-\mathrm{MCP}+100 \mathrm{ppm} \mathrm{GA}$ & 197.4 cde & 193.7 ef & $180.0 \mathrm{fgh}$ & $125.0 \mathrm{k}$ & 90.0 no & $68.0 \mathrm{rst}$ & $142.4 \mathrm{AB}$ \\
\hline $1.5 \mathrm{ppm} 1-\mathrm{MCP}+100 \mathrm{ppm} \mathrm{GA}$ & 192.7 ef & $189.0 \mathrm{efg}$ & 189.0 efg & $126.3 \mathrm{k}$ & 89.3 no & $71.0 \mathrm{qrs}$ & $142.9 \mathrm{~A}$ \\
\hline Means & $200.2 \mathrm{~A}$ & $196.5 \mathrm{~A}$ & $173.1 \mathrm{~B}$ & $110.7 \mathrm{C}$ & $80.5 \mathrm{D}$ & $60.2 \mathrm{E}$ & \\
\hline \multicolumn{8}{|c|}{ Season 2015} \\
\hline Distilled water (control) & 198.7 cde & 195.0 def & $138.0 \mathrm{n}$ & $81.7 \quad v-y$ & $51.7 \mathrm{z}$ & $44.7 \mathrm{z}$ & $118.3 \mathrm{D}$ \\
\hline $1 \mathrm{ppm} 1-\mathrm{MCP}$ & $181.4 \mathrm{f}-\mathrm{i}$ & 177.7 hij & $141.3 \mathrm{mn}$ & 114.7 op & $100.3 \mathrm{p}-\mathrm{t}$ & $86.0 \mathrm{t}-\mathrm{x}$ & $133.6 \mathrm{C}$ \\
\hline $1.5 \mathrm{ppm} 1-\mathrm{MCP}$ & 194.4 d-g & $190.7 \mathrm{e}-\mathrm{i}$ & $161.7 \mathrm{kl}$ & $106.0 \mathrm{pq}$ & $91.3 \mathrm{q}-\mathrm{v}$ & 74.7 wxy & $136.5 \mathrm{C}$ \\
\hline $50 \mathrm{ppm} \mathrm{GA}$ & $212.0 \mathrm{abc}$ & $208.3 \mathrm{bcd}$ & 127.0 no & $101.0 \mathrm{p}-\mathrm{t}$ & $94.7 \quad \mathrm{q}-\mathrm{v}$ & $66.0 \mathrm{y}$ & $134.8 \mathrm{C}$ \\
\hline $100 \mathrm{ppm} \mathrm{GA} 3$ & 196.0 def & $192.3 \mathrm{e}-\mathrm{h}$ & $153.3 \mathrm{~lm}$ & $103.0 \mathrm{p}-\mathrm{s}$ & 98.3 p-u & $67.3 \mathrm{y}$ & $135.0 \mathrm{C}$ \\
\hline $1 \mathrm{ppm} 1-\mathrm{MCP}+50 \mathrm{ppm} \mathrm{GA}$ & $217.7 \mathrm{ab}$ & $214.0 \mathrm{ab}$ & $163.7 \mathrm{jkl}$ & 112.7 op & 87.7 r-w & $69.3 \mathrm{y}$ & $144.2 \mathrm{~B}$ \\
\hline $1.5 \mathrm{ppm} 1-\mathrm{MCP}+50 \mathrm{ppm} \mathrm{GA}$ & $227.4 \mathrm{a}$ & $223.7 \mathrm{ab}$ & $178.6 \mathrm{~g}-\mathrm{j}$ & $104.3 \mathrm{pqr}$ & $89.7 \mathrm{q}-\mathrm{w}$ & $70.7 \mathrm{xy}$ & $149.1 \mathrm{AB}$ \\
\hline $1 \mathrm{ppm} 1-\mathrm{MCP}+100 \mathrm{ppm} \mathrm{GA} 3$ & $225.4 \mathrm{a}$ & $221.7 \mathrm{ab}$ & $174.9 \mathrm{ijk}$ & $102.7 \mathrm{p}-\mathrm{t}$ & 93.3 q-v & 82.0 u-y & $150.0 \mathrm{AB}$ \\
\hline $1.5 \mathrm{ppm} 1-\mathrm{MCP}+100 \mathrm{ppm} \mathrm{GA}$ & 226.4 a & $222.7 \mathrm{ab}$ & $182.9 \mathrm{e}-\mathrm{i}$ & $100.7 \mathrm{p}-\mathrm{t}$ & $93.0 \mathrm{q}-\mathrm{v}$ & $87.0 \mathrm{~s}-\mathrm{w}$ & $152.1 \mathrm{~A}$ \\
\hline Means & $208.8 \mathrm{~A}$ & $205.1 \mathrm{~A}$ & $157.9 \mathrm{~B}$ & $103.0 \mathrm{C}$ & $88.9 \mathrm{D}$ & $72.0 \mathrm{E}$ & \\
\hline
\end{tabular}

Moreover, the present results indicated that there was a positive relationship between firmness and weight loss. This observation cleared that, postharvest application with either 1-MCP or $\mathrm{GA}_{3}$ especially in combination treatments delayed ripening process, retained firmness and extended the storage life of mango fruits by reducing weight loss during storage as documented by (Jeong, 2001) on avocado fruits.

\section{Fruit skin and flesh colour}

Data illustrated in Tables 5 and 6 demonstrated that, flesh and skin colour of mango fruits represented as hue angle value (green or yellowish green, more than 90 and greenish yellow or yellow, less than 90). Fruit skin colour changed

Egypt. J. Hort. Vol. 43, No.2 (2016) 
directly from green to yellow with prolonging of cold storage period followed by shelf life during the two seasons in this research. On the other side, fruit flesh colour changed directly from yellow to orange (hue angle around 65) with prolonging of cold storage period followed by shelf life during the two seasons in this research.

TABLE 5. Effect of postharvest treatments of 1-methylcyclopropene (1-MCP) and gibberelic acid $\left(\mathrm{GA}_{3}\right)$ on changes in flesh colour represented as hue angle of 'Zibda' mango fruits during storage at $13^{\circ} \mathrm{C}$ plus 3 days shelf life at 22$28^{\circ} \mathrm{C}$

\begin{tabular}{|c|c|c|c|c|c|c|c|}
\hline \multirow{2}{*}{ Postharvest treatments } & \multicolumn{6}{|c|}{ Storage period (days) } & \multirow[b]{2}{*}{ Means } \\
\hline & $\mathbf{0}$ & 7 & 14 & 21 & 28 & 35 & \\
\hline \multicolumn{8}{|c|}{ Season 2014} \\
\hline Distilled water (control) & $79.4 \mathrm{c}-\mathrm{f}$ & $77.8 \mathrm{~d}-\mathrm{h}$ & $66.1 \mathrm{n}-\mathrm{q}$ & $63.1 \mathrm{q}$ & $55.6 \mathrm{r}$ & $52.3 \mathrm{r}$ & $65.7 \mathrm{E}$ \\
\hline 1 ppm 1-MCP & $85.2 \mathrm{ab}$ & $83.5 \mathrm{abc}$ & $77.4 \mathrm{~d}-\mathrm{h}$ & $74.6 \mathrm{f}-\mathrm{m}$ & 71.2 i-n & $71.0 \mathrm{j}-\mathrm{o}$ & $77.1 \mathrm{D}$ \\
\hline $1.5 \mathrm{ppm} 1-\mathrm{MCP}$ & $86.8 \mathrm{a}$ & $85.1 \mathrm{ab}$ & $80.8 \mathrm{~b}-\mathrm{e}$ & $75.9 \mathrm{~d}-\mathrm{j}$ & $73.1 \mathrm{~h}-\mathrm{m}$ & $67.0 \mathrm{n}-\mathrm{q}$ & $78.1 \mathrm{CD}$ \\
\hline $50 \mathrm{ppm} \mathrm{GA}$ & $88.1 \mathrm{a}$ & 86.4 a & 80.2 b-e & $75.6 \mathrm{~d}-\mathrm{k}$ & $70.4 \mathrm{k}-\mathrm{p}$ & $63.9 \mathrm{q}$ & $77.4 \mathrm{CD}$ \\
\hline $100 \mathrm{ppm} \mathrm{GA} 3$ & $88.3 \mathrm{a}$ & $86.6 \mathrm{a}$ & $79.6 \mathrm{c}-\mathrm{f}$ & $76.6 \mathrm{~d}-\mathrm{i}$ & $70.01-p$ & $63.9 \mathrm{q}$ & $77.5 \mathrm{CD}$ \\
\hline $1 \mathrm{ppm} \mathrm{1-MCP}+50$ ppm GA 3 & $88.0 \mathrm{a}$ & $86.3 \mathrm{a}$ & $83.2 \mathrm{abc}$ & 80.2 b-e & $73.1 \mathrm{~h}-\mathrm{m}$ & $65.8 \mathrm{opq}$ & $79.4 \mathrm{BC}$ \\
\hline $1.5 \mathrm{ppm} 1-\mathrm{MCP}+50 \mathrm{ppm} \mathrm{\textrm {GA } _ { 3 }}$ & $88.7 \mathrm{a}$ & $87.0 \mathrm{a}$ & $88.4 \mathrm{a}$ & $73.9 \mathrm{~g}-\mathrm{m}$ & $75.3 \mathrm{e}-1$ & 69.4 m-p & $80.5 \mathrm{~B}$ \\
\hline 1 ppm 1-MCP + 100 ppm GA 3 & 87.9 a & $86.2 \mathrm{a}$ & $87.8 \mathrm{a}$ & $79.3 \mathrm{c}-\mathrm{g}$ & $75.8 \mathrm{~d}-\mathrm{k}$ & $65.6 \mathrm{pq}$ & $80.4 \mathrm{~B}$ \\
\hline $1.5 \mathrm{ppm} 1-\mathrm{MCP}+100 \mathrm{ppm} \mathrm{GA}$ & 88.7 a & $87.1 \mathrm{a}$ & $83.9 \mathrm{abc}$ & 80.9 bcd & 80.2 b-e & 73.7 h-m & $82.4 \mathrm{~A}$ \\
\hline Means & $86.8 \mathrm{~A}$ & $85.1 \mathrm{~B}$ & $80.8 \mathrm{C}$ & 75.6 D & $71.6 \mathrm{E}$ & $65.8 \mathrm{~F}$ & \\
\hline \multicolumn{8}{|c|}{ Season 2015} \\
\hline Distilled water (control) & $69.8 \mathrm{~s}-\mathrm{v}$ & $68.1 \mathrm{uv}$ & $66.3 \mathrm{v}$ & $61.1 \mathrm{w}$ & $59.3 \mathrm{w}$ & $58.7 \mathrm{w}$ & $63.9 \mathrm{E}$ \\
\hline 1 ppm 1-MCP & $83.0 \mathrm{c}-\mathrm{j}$ & $81.3 \mathrm{e}-\mathrm{k}$ & $80.3 \mathrm{~g}-\mathrm{m}$ & $75.41-\mathrm{s}$ & $72.4 \mathrm{q}-\mathrm{u}$ & $73.1 \mathrm{o}-\mathrm{u}$ & $77.6 \mathrm{C}$ \\
\hline $1.5 \mathrm{ppm} 1-\mathrm{MCP}$ & $85.1 \mathrm{a}-\mathrm{g}$ & 83.4 b-i & $81.5 \mathrm{~d}-\mathrm{k}$ & $75.2 \mathrm{~m}-\mathrm{s}$ & $74.5 \mathrm{n}-\mathrm{t}$ & $71.9 \mathrm{q}-\mathrm{u}$ & $78.6 \mathrm{BC}$ \\
\hline $50 \mathrm{ppm} \mathrm{GA} 3$ & 78.6 i-o & $77.0 \mathrm{k}-\mathrm{r}$ & $76.2 \mathrm{k}-\mathrm{r}$ & $72.0 \mathrm{q}-\mathrm{u}$ & $71.5 \mathrm{r}-\mathrm{v}$ & 68.9 tuv & $74.1 \mathrm{D}$ \\
\hline $100 \mathrm{ppm} \mathrm{GA} 3$ & 86.0 a-f & 84.3 a-h & 79.1 h-n & $72.8 \mathrm{p}-\mathrm{u}$ & $72.5 \mathrm{q}-\mathrm{u}$ & $69.9 \mathrm{~s}-\mathrm{v}$ & $77.4 \mathrm{C}$ \\
\hline $1 \mathrm{ppm} \mathrm{1-MCP}+50 \mathrm{ppm} \mathrm{GA} 3$ & $89.3 \mathrm{a}$ & $87.7 \mathrm{abc}$ & $80.9 \mathrm{f}-1$ & 73.7 n-u & $74.2 \mathrm{n}-\mathrm{t}$ & $72.6 \mathrm{p}-\mathrm{u}$ & $79.7 \mathrm{AB}$ \\
\hline $1.5 \mathrm{ppm} \mathrm{1-MCP} \mathrm{+} 50 \mathrm{ppm} \mathrm{GA}$ & $88.2 \mathrm{abc}$ & 86.5 a-f & $81.6 \mathrm{~d}-\mathrm{k}$ & 78.7 i-o & $75.0 \mathrm{~m}-\mathrm{s}$ & $72.4 \mathrm{q}-\mathrm{u}$ & $80.4 \mathrm{AB}$ \\
\hline $1 \mathrm{ppm} 1-\mathrm{MCP}+100 \mathrm{ppm} \mathrm{GA}$ & $88.6 \mathrm{abc}$ & 86.9 a-e & 86.7 a-e & 77.6 j-q & $72.3 \mathrm{q}-\mathrm{u}$ & $72.0 \mathrm{q}-\mathrm{u}$ & $80.7 \mathrm{AB}$ \\
\hline $1.5 \mathrm{ppm} \mathrm{1-MCP}+100 \mathrm{ppm} \mathrm{GA}_{3}$ & $88.7 \mathrm{ab}$ & 87.0 a-d & $85.6 \mathrm{a}-\mathrm{g}$ & 78.2 i-p & $76.0 \mathrm{k}-\mathrm{r}$ & $75.1 \mathrm{~m}-\mathrm{s}$ & $81.8 \mathrm{~A}$ \\
\hline Means & $84.2 \mathrm{~A}$ & $82.5 \mathrm{~B}$ & $79.8 \mathrm{C}$ & $73.9 \mathrm{D}$ & $72.0 \mathrm{E}$ & $70.5 \mathrm{E}$ & \\
\hline
\end{tabular}

These results are in line with those obtained by Islam et al. (2013a) and Chutichudet et al. (2016). They mentioned that, skin colour of mango fruits changed from green and greenish yellow to yellow and pulp was changed from yellow to orange with prolonging of cold storage period. Moreover, data suggested that, all postharvest treatments significantly reduced changing rate of fruit skin and flesh colour during the two seasons in this investigation as compared to untreated fruits. In addition, fruits treated with 1-MCP in 
combination with gibberellic acid significantly had the lowest colour changing rate during the two seasons under this study. Data also approved that, there was a significant interaction among all these studded treatments during the two seasons in this research.

TABLE 6. Effect of postharvest treatments of 1-methylcyclopropene (1-MCP) and gibberelic acid $\left(\mathrm{GA}_{3}\right)$ on changes in skin colour represented as hue angle of 'Zibda' mango fruits during storage at $13^{\circ} \mathrm{C}$ plus 3 days shelf life at 22 $28^{\circ} \mathrm{C}$

\begin{tabular}{|c|c|c|c|c|c|c|c|c|c|c|}
\hline \multirow{2}{*}{ Postharvest treatments } & \multicolumn{8}{|c|}{ Storage period (days) } & \multirow{2}{*}{\multicolumn{2}{|c|}{ Means }} \\
\hline & $\mathbf{0}$ & 7 & 14 & 21 & & 88 & & 5 & & \\
\hline \multicolumn{11}{|c|}{ Season 2014} \\
\hline Distilled water ( & $121.0 \mathrm{a}-\mathrm{e}$ & $119.3 \mathrm{a}-\mathrm{e}$ & 105.2 i-n & $94.7 \mathrm{qrs}$ & 77.6 & $\mathrm{u}$ & 65.1 & $\mathrm{v}$ & $97.2 \mathrm{C}$ & C \\
\hline 1 ppm 1-MCP & 122.2 a-d & 120.5 a-e & $106.8 \mathrm{i}-\mathrm{m}$ & 98.9 n-r & 98.7 & $\mathrm{n}-\mathrm{r}$ & 96.3 & $\mathrm{p}-\mathrm{s}$ & $107.2 \mathrm{~B}$ & B \\
\hline $1.5 \mathrm{ppm} 1-\mathrm{MCP}$ & $117.1 \mathrm{c}-\mathrm{f}$ & 115.4 e-h & $110.2 \mathrm{~g}-\mathrm{k}$ & 103.7 k-o & 101.1 & $\mathrm{~m}-\mathrm{q}$ & 96.5 & $\mathrm{p}-\mathrm{s}$ & $107.3 \mathrm{~B}$ & B \\
\hline $50 \mathrm{ppm} \mathrm{GA} 3$ & $123.9 \mathrm{ab}$ & $122.3 \mathrm{a}-\mathrm{d}$ & $108.8 \mathrm{~h}-1$ & $103.9 \mathrm{k}-\mathrm{o}$ & 92.6 & rst & 90.0 & st & $106.9 \mathrm{~B}$ & B \\
\hline $100 \mathrm{ppm} \mathrm{GA} 3$ & $124.7 \mathrm{ab}$ & $123.1 \mathrm{abc}$ & $110.8 \mathrm{f}-\mathrm{j}$ & $105.8 \mathrm{i}-\mathrm{m}$ & 92.4 & rst & 87.4 & $\mathrm{t}$ & $107.4 \mathrm{~B}$ & B \\
\hline $1 \mathrm{ppm} 1-\mathrm{MCP}+50 \mathrm{ppm} \mathrm{GA}$ & $123.7 \mathrm{abc}$ & 122.0 a-e & $118.3 \mathrm{~b}-\mathrm{e}$ & $111.0 \mathrm{f}-\mathrm{j}$ & 103.9 & $\mathrm{k}-\mathrm{o}$ & 91.0 & st & $111.7 \mathrm{~A}$ & A \\
\hline $1.5 \mathrm{ppm} 1-\mathrm{MCP}+50 \mathrm{ppm} \mathrm{GA}$ & $124.8 \mathrm{ab}$ & $123.1 \mathrm{abc}$ & 118.9 b-e & $107.9 \mathrm{i}-1$ & 102.6 & $1-p$ & 96.2 & $\mathrm{p}-\mathrm{s}$ & $112.3 \mathrm{~A}$ & A \\
\hline $1 \mathrm{ppm} 1-\mathrm{MCP}+100 \mathrm{ppm} \mathrm{GA}$ & $126.1 \mathrm{a}$ & $124.4 \mathrm{ab}$ & $111.2 \mathrm{f}-\mathrm{j}$ & $111.8 \mathrm{f}-\mathrm{i}$ & 106.7 & $i-m$ & 98.4 & o-r & $113.1 \mathrm{~A}$ & A \\
\hline $1.5 \mathrm{ppm} 1-\mathrm{MCP}+100 \mathrm{ppm} \mathrm{GA}$ & $126.1 \mathrm{a}$ & $124.4 \mathrm{ab}$ & $115.6 \mathrm{~d}-\mathrm{g}$ & $111.8 \mathrm{f}-\mathrm{i}$ & 104.7 & j-o & 100.5 & $\mathrm{~m}-\mathrm{q}$ & $113.9 \mathrm{~A}$ & $\Delta$ \\
\hline Means & $123.3 \mathrm{~A}$ & $121.6 \mathrm{~A}$ & $111.8 \mathrm{~B}$ & $105.5 \mathrm{C}$ & 97.8 & $\mathrm{D}$ & 91.3 & $\mathrm{E}$ & & \\
\hline \multicolumn{11}{|c|}{ Season 2015} \\
\hline Dis & 104.2 h-l & $102.5 \mathrm{i}-\mathrm{m}$ & $92.1 \quad \mathrm{o}-\mathrm{v}$ & 89.8 q-w & 83.5 & wxy & 67.3 & $\mathrm{z}$ & $89.9 \quad \mathrm{C}$ & $\overline{\mathrm{C}}$ \\
\hline $1 \mathrm{ppm}$ & 118.5 a-f & $116.9 \mathrm{~b}-\mathrm{f}$ & $103.6 \mathrm{i}-1$ & 96.8 l-r & 94.3 & $n-\mathrm{t}$ & 84.9 & $v-y$ & $102.5 \mathrm{~B}$ & B \\
\hline $1.5 \mathrm{ppm} 1-\mathrm{MCP}$ & 121.0 a-d & 119.3 a-e & 113.2 efg & $98.0 \quad \mathrm{k}-\mathrm{p}$ & 90.9 & $\mathrm{p}-\mathrm{w}$ & 85.7 & $\mathrm{u}-\mathrm{y}$ & $104.7 \mathrm{~B}$ & B \\
\hline & 121.3 a-d & 119.6 a-e & $111.1 \mathrm{fgh}$ & 94.8 n-t & 88.2 & $\mathrm{~s}-\mathrm{x}$ & 80.7 & xy & $102.6 \mathrm{~B}$ & B \\
\hline $100 \mathrm{ppm} \mathrm{GA} 3$ & 116.7 b-f & $115.0 \mathrm{def}$ & 104.9 h-k & $101.3 \mathrm{i}-\mathrm{n}$ & 92.8 & $\mathrm{o}-\mathrm{u}$ & 80.3 & $\mathrm{y}$ & $101.8 \mathrm{~B}$ & B \\
\hline $1 \mathrm{ppm} 1-\mathrm{MCP}+50 \mathrm{ppm} \mathrm{GA}$ & $123.6 \mathrm{abc}$ & $122.0 \mathrm{a}-\mathrm{d}$ & $116.7 \mathrm{c}-\mathrm{f}$ & $101.5 \mathrm{i}-\mathrm{n}$ & 95.1 & $\mathrm{~m}-\mathrm{s}$ & 88.1 & s-x & $107.8 \mathrm{~A}$ & A \\
\hline $1.5 \mathrm{ppm} 1-\mathrm{MCP}+50 \mathrm{ppm} \mathrm{GA}{ }_{3}$ & $124.5 \mathrm{ab}$ & $122.9 \mathrm{abc}$ & $106.6 \mathrm{~g}-\mathrm{j}$ & $97.4 \quad \mathrm{k}-\mathrm{q}$ & 87.1 & $\mathrm{t}-\mathrm{y}$ & 84.8 & $v-y$ & 103.9 B & B \\
\hline $1 \mathrm{ppm} 1-\mathrm{MCP}+100 \mathrm{ppm} \mathrm{GA}$ & $124.7 \mathrm{a}$ & $123.0 \mathrm{abc}$ & $116.5 \mathrm{c}-\mathrm{f}$ & $103.5 \mathrm{i}-1$ & 94.8 & $\mathrm{n}-\mathrm{t}$ & 89.6 & $\mathrm{r}-\mathrm{w}$ & $108.7 \mathrm{~A}$ & A \\
\hline $1.5 \mathrm{ppm} \mathrm{1-MCP}+100 \mathrm{ppm} \mathrm{GA}_{3}$ & $124.8 \mathrm{a}$ & $123.1 \mathrm{abc}$ & $116.8 \mathrm{~b}-\mathrm{f}$ & 107.1 ghi & 99.3 & $\mathrm{j}-\mathrm{o}$ & 84.6 & $v-y$ & $109.3 \mathrm{~A}$ & \\
\hline Means & $119.9 \mathrm{~A}$ & $118.3 \mathrm{~A}$ & $109.1 \mathrm{~B}$ & $98.9 \mathrm{C}$ & 91.8 & $\mathrm{D}$ & 82.9 & $\mathrm{E}$ & & \\
\hline
\end{tabular}

Means followed by the same letters within postharvest treatments, storage periods and their interactions in each season are not significantly different at level $P \leq 0.05$ according to DMRT.

The duration of the exposure to 1-MCP and $\mathrm{GA}_{3}$ dipping were 24 hours and 10 minutes, respectively.

These results were confirmed by studies of (Pongprasert \& Srilaong, 2014) on bananas and (Chutichudet et al., 2016) on mangoes. They reported that, postharvest treatment of 1-MCP prevented or delayed chlorophyll degradation and retained the highest hue angle value in comparison to control during storage. In addition, these results are in line with those obtained by (Islam et al., 2013a), who illustrated that mango fruits treated with $\mathrm{GA}_{3}$ delayed colour development and retarding chlorophyll degradation in the peel as compared to untreated fruits during storage.

Egypt. J. Hort. Vol. 43, No.2 (2016) 


\section{Chemical characteristics}

Fruit contents of total soluble solids (TSS), titratable acidity (TA), TSS/TA ratio and ascorbic acid (AsA)

Data shown in Tables 7, 8, 9 and 10 clearly indicated that, mango fruit contents of TSS and TSS/TA ratio gradually and significantly increased with the advancement of cold storage period followed by shelf life, while TA and AsA gradually and significantly decreased with prolonging of storage period during the two seasons under this investigation.

These results are in agreements with the previous reports of Abu-Goukh \& Abu-Sarra (1993) and Islam et al. (2013a). They mentioned that, AsA declined rapidly during storage of mangoes. Moreover, our results wee confirmed by those obtained by Bashir \& Abu-Goukh (2003) on guava fruits and Abd El-khalek (2012) and El-Abbasy et al. (2013) on 'Eureka' lemon fruits.

TABLE 7. Effect of postharvest treatments of 1-methylcyclopropene (1-MCP) and gibberelic acid $\left(\mathrm{GA}_{3}\right)$ on changes in AsA $(\mathrm{mg} / 100 \mathrm{ml}$ juice) of 'Zibda' mango fruits during storage at $13^{\circ} \mathrm{C}$ plus 3 days shelf life at $22-28^{\circ} \mathrm{C}$

\begin{tabular}{|c|c|c|c|c|c|c|c|}
\hline \multirow{2}{*}{ Postharvest treatments } & \multicolumn{6}{|c|}{ Storage period (days) } & \multirow[b]{2}{*}{ Means } \\
\hline & $\mathbf{0}$ & 7 & 14 & 21 & 28 & 35 & \\
\hline \multicolumn{8}{|c|}{ Season 2014} \\
\hline Distilled water (control) & $55.42 \mathrm{a}-\mathrm{d}$ & 50.73 efg & $42.40 \mathrm{k}-\mathrm{o}$ & 40.17 op & $20.77 \mathrm{t}$ & $16.93 \mathrm{u}$ & $37.74 \mathrm{E}$ \\
\hline 1 ppm 1-MCP & $58.29 \mathrm{a}$ & $53.60 \mathrm{~b}-\mathrm{e}$ & $42.47 \mathrm{k}-\mathrm{o}$ & $42.64 \mathrm{j}-\mathrm{o}$ & $41.41 \mathrm{~m}-\mathrm{p}$ & 31.76 qrs & $45.03 \mathrm{BC}$ \\
\hline 1.5 ppm 1-MCP & $56.09 \mathrm{abc}$ & 51.40 ef & $48.47 \mathrm{fgh}$ & $44.97 \mathrm{i}-1$ & $34.71 \mathrm{qr}$ & 33.97 qrs & $44.94 \mathrm{BC}$ \\
\hline $50 \mathrm{ppm} \mathrm{GA}_{3}$ & $56.89 \mathrm{ab}$ & $52.20 \mathrm{e}$ & $42.201-\mathrm{o}$ & 41.00 nop & $35.07 \mathrm{q}$ & $30.93 \mathrm{~s}$ & $43.05 \mathrm{D}$ \\
\hline $100 \mathrm{ppm} \mathrm{GA} 3$ & 57.69 a & 53.00 cde & $43.00 \mathrm{j}-\mathrm{o}$ & $43.83 \mathrm{j}-\mathrm{n}$ & 33.94 qrs & $31.43 \mathrm{rs}$ & $43.82 \mathrm{CD}$ \\
\hline $1 \mathrm{ppm} \mathrm{1-MCP}+50$ ppm GA 3 & $56.69 \mathrm{ab}$ & $52.00 \mathrm{e}$ & 47.85 ghi & 44.81 i-m & $38.59 \mathrm{p}$ & $32.11 \mathrm{qrs}$ & $45.34 \mathrm{~B}$ \\
\hline $1.5 \mathrm{ppm} \mathrm{1-MCP} \mathrm{+} 50 \mathrm{ppm} \mathrm{GA}_{3}$ & $56.29 \mathrm{ab}$ & $51.60 \mathrm{e}$ & 47.88 ghi & $45.81 \mathrm{~h}-\mathrm{k}$ & $42.51 \mathrm{k}-\mathrm{o}$ & $32.49 \mathrm{qrs}$ & $46.10 \mathrm{AB}$ \\
\hline $1 \mathrm{ppm} 1-\mathrm{MCP}+100 \mathrm{ppm} \mathrm{GA}$ & $56.69 \mathrm{ab}$ & $52.00 \mathrm{e}$ & $47.82 \mathrm{ghi}$ & $45.97 \mathrm{hij}$ & $43.87 \mathrm{j}-\mathrm{n}$ & $33.85 \mathrm{qrs}$ & $46.70 \mathrm{~A}$ \\
\hline $1.5 \mathrm{ppm} 1-\mathrm{MCP}+100 \mathrm{ppm} \mathrm{GA}_{3}$ & $57.49 \mathrm{a}$ & $52.80 \mathrm{de}$ & $47.45 \mathrm{hi}$ & $45.14 \mathrm{~h}-1$ & $44.54 \mathrm{i}-\mathrm{m}$ & $34.52 \mathrm{qr}$ & $46.99 \mathrm{~A}$ \\
\hline Means & $56.84 \mathrm{~A}$ & $52.15 \mathrm{~B}$ & $45.50 \mathrm{C}$ & $43.82 \mathrm{D}$ & $37.27 \mathrm{E}$ & $30.89 \mathrm{~F}$ & \\
\hline \multicolumn{8}{|c|}{ Season 2015} \\
\hline Distilled water (control) & $51.52 \mathrm{f}-\mathrm{j}$ & 46.83 h-n & $37.27 \mathrm{~s}-\mathrm{v}$ & $38.03 \mathrm{r}-\mathrm{u}$ & $28.17 \mathrm{xy}$ & $22.10 \mathrm{z}$ & $37.32 \mathrm{E}$ \\
\hline 1 ppm 1-MCP & $58.22 \mathrm{cde}$ & 53.53 efg & 46.20 i-o & $43.27 \mathrm{k}-\mathrm{r}$ & $41.07 \mathrm{n}-\mathrm{t}$ & $30.80 \mathrm{wx}$ & $45.52 \mathrm{BC}$ \\
\hline $1.5 \mathrm{ppm} \mathrm{1-MCP}$ & 56.89 def & $52.20 \mathrm{fgh}$ & $46.57 \mathrm{~h}-\mathrm{o}$ & 43.63 k-r & $39.23 \mathrm{p}-\mathrm{u}$ & 30.83 wx & $44.89 \mathrm{C}$ \\
\hline $50 \mathrm{ppm} \mathrm{GA} 3$ & $51.89 \mathrm{f}-\mathrm{i}$ & $47.20 \mathrm{~h}-\mathrm{m}$ & 46.20 i-o & $42.901-\mathrm{s}$ & 36.30 tuv & $24.57 \mathrm{yz}$ & $41.51 \mathrm{D}$ \\
\hline $100 \mathrm{ppm} \mathrm{GA} 3$ & $48.59 \mathrm{~g}-1$ & $43.90 \mathrm{k}-\mathrm{q}$ & 45.83 j-o & $44.23 \mathrm{k}-\mathrm{p}$ & $39.23 \mathrm{p}-\mathrm{u}$ & $24.93 \mathrm{yz}$ & $41.12 \mathrm{D}$ \\
\hline $1 \mathrm{ppm} \mathrm{1-MCP}+50$ ppm GA 3 & $53.82 \mathrm{efg}$ & $49.13 \mathrm{~g}-\mathrm{k}$ & $46.57 \mathrm{~h}-\mathrm{o}$ & $44.73 \mathrm{k}-\mathrm{p}$ & 40.70 o-t & $32.00 \mathrm{vwx}$ & $44.49 \mathrm{C}$ \\
\hline 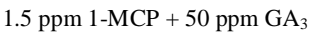 & $63.46 \mathrm{abc}$ & 58.77 cde & $43.63 \mathrm{k}-\mathrm{r}$ & $41.07 \mathrm{n}-\mathrm{t}$ & $38.13 \mathrm{q}-\mathrm{u}$ & $32.47 \mathrm{vwx}$ & $46.26 \mathrm{BC}$ \\
\hline $1 \mathrm{ppm} 1-\mathrm{MCP}+100 \mathrm{ppm} \mathrm{GA}$ & $66.02 \mathrm{ab}$ & $61.33 \mathrm{bcd}$ & $46.93 \mathrm{~h}-\mathrm{n}$ & $42.17 \mathrm{~m}-\mathrm{s}$ & $38.87 \mathrm{p}-\mathrm{u}$ & 29.70 wxy & $47.50 \mathrm{AB}$ \\
\hline $1.5 \mathrm{ppm} 1-\mathrm{MCP}+100 \mathrm{ppm} \mathrm{GA}$ & $67.12 \mathrm{a}$ & $62.43 \mathrm{abc}$ & $44.73 \mathrm{k}-\mathrm{p}$ & $42.17 \mathrm{~m}-\mathrm{s}$ & $39.23 \mathrm{p}-\mathrm{u}$ & 34.83 uvw & $48.42 \mathrm{~A}$ \\
\hline Means & $57.50 \mathrm{~A}$ & $52.81 \mathrm{~B}$ & $44.88 \mathrm{C}$ & $42.47 \mathrm{D}$ & $37.88 \mathrm{E}$ & $29.14 \mathrm{~F}$ & \\
\hline
\end{tabular}


Data in the present study also revealed that, all postharvest treatments significantly decreased loss of fruit contents in TA and AsA as well as slowed an increase of fruit contents in TSS and TSS/TA ratio during storage in both seasons under this investigation as compared to untreated fruits. Moreover, mango fruits treated with 1-MCP in combination with gibberellic acid significantly had the lowest changing rate in all these characteristics during the two seasons under this study. On contrary, in untreated fruits (control), fruit content of TSS was increased till 21 days of cold storage period followed by three days shelf life, then decreased till end of storage period as compared to the all postharvest treatment, that showed an increase in TSS till end of the experiment. Data also mentioned that, there was a significant interaction among all these studded treatments during the two seasons in this research.

TABLE 8. Effect of postharvest treatments of 1-methylcyclopropene (1-MCP) and gibberelic acid $\left(\mathrm{GA}_{3}\right)$ on changes in TSS ( $\left.{ }^{\circ} \mathrm{Brix}\right)$ of 'Zibda' mango fruits during storage at $13^{\circ} \mathrm{C}$ plus 3 days shelf life at $22-28^{\circ} \mathrm{C}$

\begin{tabular}{|c|c|c|c|c|c|c|c|c|}
\hline \multirow{2}{*}{ Postharvest treatments } & \multicolumn{7}{|c|}{ Storage period (days) } & \multirow[b]{2}{*}{ Means } \\
\hline & & $\mathbf{0}$ & 7 & 14 & 21 & 28 & 35 & \\
\hline \multicolumn{9}{|c|}{ Season 2014} \\
\hline Distilled water (c & 11.00 & uvw & $12.40 \mathrm{r}$ & $15.87 \mathrm{~lm}$ & $21.77 \mathrm{a}$ & $19.30 \mathrm{bc}$ & $17.17 \mathrm{hij}$ & $16.25 \mathrm{~A}$ \\
\hline 1 ppm 1-MCP & 10.73 & vwx & $12.13 \mathrm{rs}$ & 14.73 no & 15.971 & 17.80 e-h & $19.27 \mathrm{bc}$ & $15.11 \mathrm{~B}$ \\
\hline $1.5 \mathrm{ppm} 1-\mathrm{MCP}$ & 10.47 & wxy & $11.87 \mathrm{rst}$ & 14.50 op & $15.30 \mathrm{mn}$ & $17.53 \mathrm{f}-\mathrm{i}$ & $18.87 \mathrm{~cd}$ & $14.76 \mathrm{C}$ \\
\hline $50 \mathrm{ppm} \mathrm{GA}$ & 10.73 & vwx & $12.13 \mathrm{rs}$ & 14.93 no & $16.23 \mathrm{kl}$ & $18.37 \mathrm{de}$ & $19.80 \mathrm{~b}$ & $15.37 \mathrm{~B}$ \\
\hline $100 \mathrm{ppm} \mathrm{GA} 3$ & 10.70 & vwx & $12.10 \mathrm{rs}$ & 14.90 no & 16.071 & 18.10 ef & $19.67 \mathrm{~b}$ & $15.26 \mathrm{~B}$ \\
\hline $1 \mathrm{ppm} 1-\mathrm{MCP}+50 \mathrm{ppm} \mathrm{GA} 3$ & 10.23 & xy & $11.63 \mathrm{stu}$ & $14.07 \mathrm{pq}$ & 15.00 no & $17.40 \mathrm{~g}-\mathrm{j}$ & 18.00 efg & $14.39 \mathrm{D}$ \\
\hline $1.5 \mathrm{ppm} 1-\mathrm{MCP}+50 \mathrm{ppm} \mathrm{\textrm {GA } _ { 3 }}$ & 10.10 & $x y$ & $11.50 \mathrm{stu}$ & $13.97 \mathrm{pq}$ & 15.00 no & $16.77 \mathrm{jk}$ & $17.37 \mathrm{~g}-\mathrm{j}$ & $14.12 \mathrm{E}$ \\
\hline $1 \mathrm{ppm} \mathrm{1-MCP}+100$ ppm GA 3 & 9.93 & $\mathrm{y}$ & 11.33 tuv & $13.73 \mathrm{q}$ & 14.83 no & $16.33 \mathrm{kl}$ & $17.27 \mathrm{hij}$ & $13.90 \mathrm{EF}$ \\
\hline $1.5 \mathrm{ppm} 1-\mathrm{MCP}+100 \mathrm{ppm} \mathrm{GA}_{3}$ & 9.83 & $\mathrm{y}$ & 11.23 tuv & $13.67 \mathrm{q}$ & 14.77 no & 15.971 & $16.87 \mathrm{ijk}$ & $13.72 \mathrm{~F}$ \\
\hline Means & 10.41 & $\mathrm{~F}$ & $11.81 \mathrm{E}$ & $14.49 \mathrm{D}$ & $16.10 \mathrm{C}$ & $17.51 \mathrm{~B}$ & $18.25 \mathrm{~A}$ & \\
\hline \multicolumn{9}{|c|}{ Season 2015} \\
\hline Distilled water & 11.20 & uv & $12.60 \mathrm{~s}$ & $15.57 \mathrm{mn}$ & $20.93 \mathrm{a}$ & $18.63 \mathrm{~b}$ & $17.00 \mathrm{f}-\mathrm{j}$ & $15.99 \mathrm{~A}$ \\
\hline 1 ppm 1-MCP & 10.37 & wx & $11.77 \mathrm{tu}$ & $14.40 \mathrm{pq}$ & $16.27 \mathrm{kl}$ & $17.57 \mathrm{~d}-\mathrm{h}$ & $17.90 \mathrm{cde}$ & $14.71 \mathrm{C}$ \\
\hline $1.5 \mathrm{ppm} 1-\mathrm{MCP}$ & 10.37 & wx & 11.77 tu & $14.37 \mathrm{pq}$ & $16.10 \mathrm{~lm}$ & 17.40 e-h & $17.73 \mathrm{def}$ & $14.62 \mathrm{C}$ \\
\hline $50 \mathrm{ppm} \mathrm{GA} 3$ & 11.03 & $\mathrm{v}$ & $12.43 \mathrm{~s}$ & 14.77 op & $16.90 \mathrm{~h}-\mathrm{k}$ & $17.70 \mathrm{~d}-\mathrm{g}$ & $18.47 \mathrm{bc}$ & $15.22 \mathrm{~B}$ \\
\hline $100 \mathrm{ppm} \mathrm{GA} 3$ & 10.83 & vw & $12.23 \mathrm{st}$ & 14.70 op & $16.50 \mathrm{i}-1$ & $17.60 \mathrm{~d}-\mathrm{h}$ & $18.20 \mathrm{bcd}$ & $15.01 \mathrm{~B}$ \\
\hline $1 \mathrm{ppm} 1-\mathrm{MCP}+50 \mathrm{ppm} \mathrm{GA}$ & 10.03 & $\mathrm{x}$ & $11.43 \mathrm{uv}$ & $14.27 \mathrm{pq}$ & $15.50 \mathrm{mn}$ & $17.27 \mathrm{e}-\mathrm{h}$ & $17.67 \mathrm{~d}-\mathrm{g}$ & $14.36 \mathrm{D}$ \\
\hline $1.5 \mathrm{ppm} 1-\mathrm{MCP}+50 \mathrm{ppm} \mathrm{GA}$ & 9.97 & $\mathrm{x}$ & $11.37 \mathrm{uv}$ & $13.83 \mathrm{qr}$ & 15.27 no & $16.97 \mathrm{~g}-\mathrm{k}$ & $17.47 \mathrm{e}-\mathrm{h}$ & $14.15 \mathrm{D}$ \\
\hline $1 \mathrm{ppm} \mathrm{1-MCP}+100 \mathrm{ppm} \mathrm{GA}_{3}$ & 9.90 & $\mathrm{x}$ & $11.30 \mathrm{uv}$ & $13.57 \mathrm{r}$ & 14.97 nop & $16.43 \mathrm{jkl}$ & $17.17 \mathrm{e}-\mathrm{i}$ & $13.89 \mathrm{E}$ \\
\hline $1.5 \mathrm{ppm} 1-\mathrm{MCP}+100 \mathrm{ppm} \mathrm{GA}_{3}$ & 9.73 & $\mathrm{x}$ & $11.13 \mathrm{uv}$ & $13.30 \mathrm{r}$ & 14.67 op & $16.37 \mathrm{jkl}$ & $16.97 \mathrm{~g}-\mathrm{k}$ & $13.70 \mathrm{E}$ \\
\hline Means & 10.38 & $\mathrm{~F}$ & $11.78 \mathrm{E}$ & $14.31 \mathrm{D}$ & $16.35 \mathrm{C}$ & $17.33 \mathrm{~B}$ & $17.62 \mathrm{~A}$ & \\
\hline
\end{tabular}

Means followed by the same letters within postharvest treatments, storage periods and their interactions in each season are not significantly different at level $P \leq 0.05$ according to DMRT. The duration of the exposure to $1-\mathrm{MCP}$ and $\mathrm{GA}_{3}$ dipping were 24 hours and 10 minutes, respectively.

Egypt. J. Hort. Vol. 43, No.2 (2016) 
Citric acid is a respiratory substrate and its consumption in respiration increased with the progress of storage period, whereby could be used as an organic substrate in the respiration process (Chandra et al., 1994 and DoreyappyGowda \& Huddar, 2001). The loss of fruit content in acidity was attributed to the conversion of citric acid into sugars and their further utilization in metabolic process of the fruit (Doreyappy-Gowda \& Huddar, 2001 and Rathore et al., 2007). They also added that, this might be responsible for the observed decreasing in acidity and increment in TSS during storage.

TABLE 9. Effect of postharvest treatments of 1-methylcyclopropene (1-MCP) and gibberelic acid $\left(\mathrm{GA}_{3}\right)$ on changes in $\mathrm{TA}$ ( $\mathrm{g}$ citric acid/100 ml juice) of 'Zibda' mango fruits during storage at $13^{\circ} \mathrm{C}$ plus 3 days shelf life at 22 $28^{\circ} \mathrm{C}$

\begin{tabular}{|c|c|c|c|c|c|c|c|c|c|}
\hline \multirow{2}{*}{ Postharvest treatments } & \multicolumn{8}{|c|}{ Storage period (days) } & \multirow[b]{2}{*}{ Means } \\
\hline & $\mathbf{0}$ & 7 & 14 & & 21 & & 28 & 35 & \\
\hline \multicolumn{10}{|c|}{ Season 2014} \\
\hline Distilled water (control) & $1.55 \mathrm{a}$ & $1.23 \mathrm{~b}$ & 0.91 ef & 0.66 & $\mathrm{jkl}$ & 0.53 & op & $0.52 \mathrm{p}$ & $0.90 \mathrm{D}$ \\
\hline 1 ppm 1-MCP & $1.58 \mathrm{a}$ & $1.25 \mathrm{~b}$ & $1.01 \mathrm{~cd}$ & 0.82 & gh & 0.69 & ijk & $0.57 \mathrm{~m}-\mathrm{p}$ & $0.99 \mathrm{BC}$ \\
\hline $1.5 \mathrm{ppm} 1-\mathrm{MCP}$ & $1.53 \mathrm{a}$ & $1.20 \mathrm{~b}$ & $1.03 \mathrm{~cd}$ & 0.86 & fg & 0.68 & $i-1$ & $0.62 \mathrm{k}-\mathrm{n}$ & $0.99 \mathrm{C}$ \\
\hline $50 \mathrm{ppm} \mathrm{GA} 3$ & $1.57 \mathrm{a}$ & $1.24 \mathrm{~b}$ & $1.02 \mathrm{~cd}$ & 0.85 & fg & 0.65 & $\mathrm{j}-\mathrm{m}$ & 0.56 nop & $0.98 \mathrm{C}$ \\
\hline $100 \mathrm{ppm} \mathrm{GA} 3$ & $1.57 \mathrm{a}$ & $1.24 \mathrm{~b}$ & $1.01 \mathrm{~cd}$ & 0.95 & de & 0.72 & ij & $0.63 \mathrm{k}-\mathrm{n}$ & $1.02 \mathrm{AB}$ \\
\hline $1 \mathrm{ppm} 1-\mathrm{MCP}+50 \mathrm{ppm} \mathrm{GA}$ & $1.53 \mathrm{a}$ & $1.20 \mathrm{~b}$ & $1.02 \mathrm{~cd}$ & 0.91 & ef & 0.67 & $\mathrm{jkl}$ & $0.63 \mathrm{k}-\mathrm{n}$ & $0.99 \mathrm{BC}$ \\
\hline $1.5 \mathrm{ppm} 1-\mathrm{MCP}+50 \mathrm{ppm} \mathrm{GA}$ & $1.53 \mathrm{a}$ & $1.20 \mathrm{~b}$ & $1.07 \mathrm{c}$ & 0.82 & gh & 0.67 & $\mathrm{jkl}$ & $0.601-0$ & $0.98 \mathrm{C}$ \\
\hline $1 \mathrm{ppm} 1-\mathrm{MCP}+100 \mathrm{ppm} \mathrm{GA}$ & $1.54 \mathrm{a}$ & $1.22 \mathrm{~b}$ & $1.06 \mathrm{c}$ & 0.89 & efg & 0.72 & $\mathrm{ij}$ & $0.66 \mathrm{jkl}$ & $1.01 \mathrm{ABC}$ \\
\hline $1.5 \mathrm{ppm} 1-\mathrm{MCP}+100 \mathrm{ppm} \mathrm{GA}_{3}$ & $1.58 \mathrm{a}$ & $1.25 \mathrm{~b}$ & $1.03 \mathrm{~cd}$ & 0.97 & de & 0.76 & hi & $0.67 \mathrm{jkl}$ & $1.04 \mathrm{~A}$ \\
\hline Means & $1.55 \mathrm{~A}$ & $1.22 \mathrm{~B}$ & $1.02 \mathrm{C}$ & 0.86 & $\mathrm{D}$ & 0.68 & $\mathrm{E}$ & $0.60 \mathrm{~F}$ & \\
\hline \multicolumn{10}{|c|}{ Season 2015} \\
\hline Distilled water (control) & $1.63 \mathrm{c}$ & $1.30 \mathrm{~g}$ & $1.01 \mathrm{i}$ & 0.75 & $\mathrm{~m}$ & 0.49 & o & $0.41 \mathrm{p}$ & $0.93 \mathrm{C}$ \\
\hline 1 ppm 1-MCP & $1.72 \mathrm{~b}$ & $1.40 \mathrm{f}$ & $0.96 \mathrm{ijk}$ & 0.78 & $\mathrm{~m}$ & 0.62 & $\mathrm{n}$ & 0.46 op & $0.99 \mathrm{~A}$ \\
\hline $1.5 \mathrm{ppm} 1-\mathrm{MCP}$ & $1.78 \mathrm{a}$ & $1.46 \mathrm{e}$ & $0.93 \mathrm{jk}$ & 0.77 & $\mathrm{~m}$ & 0.61 & $\mathrm{n}$ & 0.48 o & $1.00 \mathrm{~A}$ \\
\hline $50 \mathrm{ppm} \mathrm{GA}_{3}$ & $1.54 \mathrm{~d}$ & $1.22 \mathrm{~h}$ & $0.97 \mathrm{ij}$ & 0.84 & 1 & 0.62 & $\mathrm{n}$ & 0.50 o & $0.95 \mathrm{BC}$ \\
\hline $100 \mathrm{ppm} \mathrm{GA} 3$ & $1.66 \mathrm{c}$ & $1.33 \mathrm{~g}$ & $0.96 \mathrm{ijk}$ & 0.74 & $\mathrm{~m}$ & 0.62 & $\mathrm{n}$ & 0.45 op & $0.96 \mathrm{~B}$ \\
\hline $1 \mathrm{ppm} 1-\mathrm{MCP}+50 \mathrm{ppm} \mathrm{GA}$ & $1.78 \mathrm{a}$ & $1.46 \mathrm{e}$ & $0.91 \mathrm{k}$ & 0.77 & $\mathrm{~m}$ & 0.59 & $\mathrm{n}$ & 0.51 o & $1.00 \mathrm{~A}$ \\
\hline $1.5 \mathrm{ppm} 1-\mathrm{MCP}+50 \mathrm{ppm} \mathrm{GA}$ & $1.80 \mathrm{a}$ & $1.47 \mathrm{e}$ & $0.93 \mathrm{jk}$ & 0.76 & $\mathrm{~m}$ & 0.60 & $\mathrm{n}$ & 0.45 op & $1.00 \mathrm{~A}$ \\
\hline $1 \mathrm{ppm} 1-\mathrm{MCP}+100 \mathrm{ppm} \mathrm{GA}$ & $1.80 \mathrm{a}$ & $1.47 \mathrm{e}$ & $0.93 \mathrm{jk}$ & 0.77 & $\mathrm{~m}$ & 0.59 & $\mathrm{n}$ & 0.49 o & $1.01 \mathrm{~A}$ \\
\hline $1.5 \mathrm{ppm} 1-\mathrm{MCP}+100 \mathrm{ppm} \mathrm{GA}$ & $1.78 \mathrm{a}$ & $1.46 \mathrm{e}$ & $0.94 \mathrm{jk}$ & 0.72 & $\mathrm{~m}$ & 0.58 & $\mathrm{n}$ & 0.46 op & $0.99 \mathrm{~A}$ \\
\hline Means & $1.72 \mathrm{~A}$ & $1.39 \mathrm{~B}$ & $0.95 \mathrm{C}$ & 0.77 & $\mathrm{D}$ & 0.59 & E & $0.47 \mathrm{~F}$ & \\
\hline
\end{tabular}

Means followed by the same letters within postharvest treatments, storage periods and their interactions in each season are not significantly different at level $P \leq 0.05$ according to DMRT. The duration of the exposure to $1-\mathrm{MCP}$ and $\mathrm{GA}_{3}$ dipping were 24 hours and 10 minutes, respectively.

In addition, the loss of fruit content in AsA with the progress of storage period could be attributed to rapid conversion of L-ascorbic acid into dihydroascorbic acid in the presence of L-ascorbic acid oxidase (Smirnoff, 1996 and Zumreoglu-Karan, 2006). 
The available results in this study were supported by the findings of Laamim et al. (2005) on mandarins, Win et al. (2006) on limes and Abd El-Khalek (2012) and El-Abbasy et al. (2013) on 'Eureka' lemon fruits. They mentioned that, postharvest treatments of 1-MCP decreased loss of fruit content in TA as compared to control during storage periods. Moreover, these results fall in line with the findings of Wahdan et al. (2011) and Islam et al. (2013b) on mango fruits. They claimed that, postharvest treatments of $\mathrm{GA}_{3}$ maintained fruit content of TA in comparison to untreated fruits (control) during storage period.

TABLE 10. Effect of postharvest treatments of 1-methylcyclopropene (1-MCP) and gibberelic acid $\left(\mathrm{GA}_{3}\right)$ on changes in TSS/TA ratio of 'Zibda' mango fruits during storage at $13^{\circ} \mathrm{C}$ plus 3 days shelf life at $22-28^{\circ} \mathrm{C}$

\begin{tabular}{|c|c|c|c|c|c|c|c|c|}
\hline \multirow{2}{*}{ Postharvest treatments } & \multicolumn{7}{|c|}{ Storage period (days) } & \multirow[b]{2}{*}{ Means } \\
\hline & $\mathbf{0}$ & 7 & & 14 & 21 & 28 & 35 & \\
\hline \multicolumn{9}{|c|}{ Season 2014} \\
\hline Distilled water (control) & $7.12 \mathrm{q}$ & 10.20 & $\mathrm{p}$ & $17.51 \mathrm{kl}$ & $33.16 \mathrm{bc}$ & $36.20 \mathrm{a}$ & $33.23 \mathrm{bc}$ & $22.90 \mathrm{~A}$ \\
\hline 1 ppm 1-MCP & $6.80 \mathrm{q}$ & 9.70 & $\mathrm{p}$ & 14.59 no & $19.58 \mathrm{ij}$ & $25.87 \mathrm{~g}$ & $33.83 \mathrm{~b}$ & $18.39 \mathrm{~B}$ \\
\hline $1.5 \mathrm{ppm} 1-\mathrm{MCP}$ & $6.86 \mathrm{q}$ & 9.90 & $\mathrm{p}$ & 14.05 no & $17.90 \mathrm{jkl}$ & $25.81 \mathrm{~g}$ & $30.50 \mathrm{de}$ & $17.50 \mathrm{C}$ \\
\hline $50 \mathrm{ppm} \mathrm{GA} 3$ & $6.86 \mathrm{q}$ & 9.81 & $\mathrm{p}$ & 14.74 no & $19.15 \mathrm{jk}$ & $28.17 \mathrm{f}$ & $35.65 \mathrm{a}$ & $19.06 \mathrm{~B}$ \\
\hline $100 \mathrm{ppm} \mathrm{GA} 3$ & $6.83 \mathrm{q}$ & 9.77 & $\mathrm{p}$ & 14.75 no & $16.95 \mathrm{~lm}$ & $25.05 \mathrm{~g}$ & $31.48 \mathrm{~cd}$ & $17.47 \mathrm{C}$ \\
\hline $1 \mathrm{ppm} 1-\mathrm{MCP}+50 \mathrm{ppm} \mathrm{GA}$ & $6.72 \mathrm{q}$ & 9.73 & $\mathrm{p}$ & 13.81 no & $16.60 \mathrm{~lm}$ & $26.06 \mathrm{~g}$ & 28.84 ef & $16.96 \mathrm{C}$ \\
\hline $1.5 \mathrm{ppm} 1-\mathrm{MCP}+50 \mathrm{ppm} \mathrm{GA}$ & $6.60 \mathrm{q}$ & 9.57 & $\mathrm{p}$ & 13.17 o & $18.29 \mathrm{jkl}$ & $25.10 \mathrm{~g}$ & 28.88 ef & $16.93 \mathrm{C}$ \\
\hline $1 \mathrm{ppm} 1-\mathrm{MCP}+100 \mathrm{ppm} \mathrm{GA}$ & $6.44 \mathrm{q}$ & 9.33 & $\mathrm{p}$ & $13.02 \mathrm{o}$ & $16.71 \mathrm{~lm}$ & $22.64 \mathrm{~h}$ & $26.40 \mathrm{~g}$ & $15.76 \mathrm{D}$ \\
\hline $1.5 \mathrm{ppm} 1-\mathrm{MCP}+100 \mathrm{ppm} \mathrm{GA}_{3}$ & $6.23 \mathrm{q}$ & 8.98 & $\mathrm{p}$ & 13.23 o & $15.27 \mathrm{mn}$ & $21.15 \mathrm{hi}$ & $25.38 \mathrm{~g}$ & $15.04 \mathrm{E}$ \\
\hline Means & $6.72 \mathrm{~F}$ & 9.67 & E & $14.32 \mathrm{D}$ & $19.29 \mathrm{C}$ & $26.23 \mathrm{~B}$ & $30.47 \mathrm{~A}$ & \\
\hline \multicolumn{9}{|c|}{ Season 2015} \\
\hline Distilled water (control) & $6.88 \mathrm{ijk}$ & 9.70 & hi & $15.44 \mathrm{~g}$ & $27.97 \mathrm{e}$ & $38.53 \mathrm{bc}$ & $41.43 \mathrm{a}$ & $23.33 \mathrm{~A}$ \\
\hline $1 \mathrm{ppm} 1-\mathrm{MCP}$ & $6.01 \mathrm{k}$ & 8.43 & $h-k$ & $14.99 \mathrm{~g}$ & $20.98 \mathrm{f}$ & $28.36 \mathrm{e}$ & $39.01 \mathrm{abc}$ & $19.63 \mathrm{BC}$ \\
\hline $1.5 \mathrm{ppm} 1-\mathrm{MCP}$ & $5.82 \mathrm{k}$ & 8.09 & $\mathrm{~h}-\mathrm{k}$ & $15.46 \mathrm{~g}$ & $20.98 \mathrm{f}$ & $28.72 \mathrm{e}$ & $37.12 \mathrm{~cd}$ & $19.36 \mathrm{BCD}$ \\
\hline $50 \mathrm{ppm} \mathrm{GA} 3$ & $7.15 \mathrm{ijk}$ & 10.23 & $\mathrm{~h}$ & $15.18 \mathrm{~g}$ & $20.09 \mathrm{f}$ & $28.37 \mathrm{e}$ & $36.68 \mathrm{~cd}$ & $19.62 \mathrm{BC}$ \\
\hline $100 \mathrm{ppm} \mathrm{GA} 3$ & $6.54 \mathrm{jk}$ & 9.22 & hij & $15.30 \mathrm{~g}$ & $22.41 \mathrm{f}$ & $28.41 \mathrm{e}$ & $40.24 \mathrm{ab}$ & $20.35 \mathrm{~B}$ \\
\hline $1 \mathrm{ppm} 1-\mathrm{MCP}+50 \mathrm{ppm} \mathrm{GA} 3$ & $5.63 \mathrm{k}$ & 7.86 & $\mathrm{~h}-\mathrm{k}$ & $15.70 \mathrm{~g}$ & $20.27 \mathrm{f}$ & $29.15 \mathrm{e}$ & $34.49 \mathrm{~d}$ & $18.85 \mathrm{CD}$ \\
\hline $1.5 \mathrm{ppm} 1-\mathrm{MCP}+50 \mathrm{ppm} \mathrm{GA} 3$ & $5.54 \mathrm{k}$ & 7.72 & $\mathrm{~h}-\mathrm{k}$ & $14.88 \mathrm{~g}$ & $20.16 \mathrm{f}$ & $28.44 \mathrm{e}$ & $38.64 \mathrm{bc}$ & $19.23 \mathrm{CD}$ \\
\hline $1 \mathrm{ppm} 1-\mathrm{MCP}+100 \mathrm{ppm} \mathrm{GA} 3$ & $5.50 \mathrm{k}$ & 7.67 & $\mathrm{~h}-\mathrm{k}$ & $14.60 \mathrm{~g}$ & $19.53 \mathrm{f}$ & $27.87 \mathrm{e}$ & $35.25 \mathrm{~d}$ & $18.40 \mathrm{D}$ \\
\hline $1.5 \mathrm{ppm} 1-\mathrm{MCP}+100 \mathrm{ppm} \mathrm{GA}$ & $5.46 \mathrm{k}$ & 7.65 & h-k & $14.28 \mathrm{~g}$ & $20.28 \mathrm{f}$ & $28.21 \mathrm{e}$ & $36.78 \mathrm{~cd}$ & $18.78 \mathrm{CD}$ \\
\hline Means & $6.06 \mathrm{~F}$ & 8.51 & $\mathrm{E}$ & $15.09 \mathrm{D}$ & $21.41 \mathrm{C}$ & $29.56 \mathrm{~B}$ & $37.74 \mathrm{~A}$ & \\
\hline
\end{tabular}

Furthermore, these results are in line with the findings of Wahdan et al. (2011), who reported that $\mathrm{GA}_{3}$ postharvest treatment reduced the deterioration rate of mango fruits content in AsA during storage. In addition, our results are in agreement with the findings of Abdalnoor (2010), who demonstrated that 1-MCP postharvest treatment decreased loss of banana fruits content in AsA during storage.

Egypt. J. Hort. Vol. 43, No.2 (2016) 
In addition, these results are in accordance with those illustrated by Abd ElKhalek (2012) and El-Abbasy et al. (2013). They mentioned that, postharvest treatment of 1-MCP significantly slowed the increase of 'Eureka' lemons contents of TSS and TSS/TA ratio as compared to control during storage. Moreover, the obtained results are in agreement with those mentioned by Ahmed \& Singh (2000). They reported that, postharvest treatment of $\mathrm{GA}_{3}$ significantly delayed mango fruit content of TSS/TA ratio in comparison to untreated fruits during storage.

\section{Conclusion}

Our results revealed that, postharvest application of 'Zibda' mangoe fruits with exposed to 1-MCP for 24 hours or dipped in $\mathrm{GA}_{3}$ solution for ten minutes especially combined treatments before storage at $13^{\circ} \mathrm{C}$ alleviated decay incidence, reduced weight loss percentage and increased marketable fruit percentage. In addition, these applications constricted the variability of fruit colour, decreased loss of fruit firmness and maintained the fruit quality for up to 5 weeks at $13^{\circ} \mathrm{C}$ and $85-90 \% \mathrm{RH}$ followed by three days at room conditions (22$28^{\circ} \mathrm{C}$ and $\left.60-75 \% \mathrm{RH}\right)$.

Author contributions: A.F. Abd El-khalek conceived of study, designed the experiment and purchased the chemicals. A.F. Abd El-khalek and A.M.R.A. Abdelaziz performed the experiment. A.F. Abd El-khalek analyzed the data and wrote the manuscript. M.A.A Mohamed revised the manuscript.

Conflicts of interest: the authors declare that there are no conflicts of interest related to the publication of this study.

\section{References}

Abd El-khalek, A.F. (2012) Reducing chilling injury of Eureka lemon fruits during cold storage. Ph.D. Thesis, Tanta University, Egypt.

Abdalnoor, I.K.S. (2010) Effect of 1-Methylcyclopropene (1-MCP) on quality and shelflife of banana fruits. M.Sc. Thesis, Khartoum University, Sudan.

Abu-Goukh, A.A. and Abu-Sarra, A.F. (1993) Compositional changes during mango fruit ripening. University of Khartoum Journal of Agricultural sciences, 1 (1), 33-51.

Ahmed, M.S. and Singh, S. (2000) Studies on extension of storage life of Amrapali mango. Orissa Journal of Horticulture, 28, 73-76.

Ali, Z.M., Chin, L.H. and Lazan, H. (2004) A comparative study on wall degrading enzymes, pectin modifications and softening during ripening of selected tropical fruits. Plant Science, 167, 317-327. 
AOAC, (2000) "Official Methods of Analysis". 17 ${ }^{\text {th }}$ ed., Association of Official Analytical Chemist, Washington, D.C, 16-20.

Bashir, H.A. and Abu-Goukh, A.A. (2003) Compositional changes during guava fruit ripening. Journal of Food Chemistry, 80, 557-563.

Ben-Yehoshua, S., Rodov, V., Fang, D.Q. and Kim, J.J. (1995) Preformed antifungal compounds of citrus fruit: effect of postharvest treatments with heat and growth regulators. Journal of Agricultural and Food Chemistry, 43, 1062-1066.

Boquete, E.J., Trinchero, G.D., Fraschina, A.A., Vilella, F. and Sozzli, G.O. (2004) Ripening of Hayward kiwifruit treated with 1-methylcyclopropene after cold storage. Postharvest Biology and Technology, 32, 57-65.

Chandra, R., Goving, S. and Basuchaudhun, P. (1994) Preharvest sprays of calcium nitrate and Alar on quality and postharvest behaviour of guava fruits. Indian Journal of Hill Farming, 7 (1), 51-56 (Hort. Abst., 66:10947).

Chutichudet, B., Chutichudet, P. and Trainoak, U. (2016) Effects of 1-MCP on external postharvest qualities and shelf life of 'Maha Chanok' mango fruit. Journal of Agricultural Science, 8(1), 68-79

Del Monte, C.F., Tadeo, J.J., Elesbao, A.R., Cunha, F.H.A. and Canto, P.M.E. (2004) Respiration rate and chemical characteristics of cold stored 'Tommy Atkins' mangoes influenced by 1-MCP and modified atmosphere packaging. Acta Horticulture, 645, 645-650.

Doreyappy-Gowda, I.N.D. and Huddar, A.G. (2001) Studies on ripening changes in mango ((Mangifera indica L.) fruits. Journal of Food Science and Technology Mysore, 38, 135-137

El-Abbasy, U.K., Mohamed, E.M., El-Aidy, A.A., Mohamed, M.A.A. and Abd Elkhalek, A.F. (2013) Impact of ascorbic acid on Eureka lemon fruits under low temperature stress. The $1^{\text {st }}$ International Conference "Food and Agriculture: New Approaches". National Research Centre, 2-4 December, 2013, Egypt. Book of Abstracts, pp. 164.

El-Otmani, M. and Coggins Jr., C.W. (1991) Growth regulator effects on retention of quality of stored citrus. Scientia Horticulturae, 45 (3-4), 261-271.

Feng, Z., Zi-juan, G. and Wen, L. (2016) Effect of gibberellin (GA) on preservation quality of Tainong mango fruits. Guangdong Agricultural Sciences, 2016-05.

Herianus, J. D., Singh, L. Z. and Tan, S. C. (2003) Aroma volatiles production during fruit ripening of Kensington Pride Mango. Postharvest Biology and Technology, 27, 323-336.

Islam, Md.K., Rafiul Islam, A.K.M., Sarkar, M.A.R., Khan, M.Z.H. and Yeasmin, S. (2013a) Changes in color and physiological components of the postharvest mango

Egypt. J. Hort. Vol. 43, No.2 (2016) 
(Mangifera indica L.) influenced by different levels of $\mathrm{GA}_{3}$. Aceh International Journal of Science and Technology, 2 (2), 70-76

Islam, Md.K., Khan, M.Z.H., Sarkar, M.A.R., Yeasmin, S., Ali, Md.K. and Uddin, M.H. (2013b) Postharvest quality of mango (mangifera indica L.) fruit affected by different levels of gibberellic acid during storage. Malaysian Journal of Analytical Sciences, 17 (3), 499-509.

Jeong, J., Huber, D. J. and Sargent, S. A. (2001) Influence of 1-methylcyclopropene (1MCP) on ripening and cell-wall matrix polysaccharides of avocado fruit (Persea americana). Postharvest Biology and Technology, 25, 241-364.

Jeong, J., Huber, D.J. and Sargent, S.A. (2003) Delay of avocado (Persea americana) fruit ripening by 1-methylcyclopropene and wax treatments. Postharvest Biology and Technology, 28, 247-257.

Kumar, P. and Singh, S. (1993) Effect of $\mathrm{GA}_{3}$ and ethrel on ripening and quality of mango cv. 'Amrapali'. Horticulture Journal, 6 (1), 19-23.

Laamim, M. Oubahou, A. A. and Benichou, M. (2005) Effect of 1-methylcyclopropene on the quality of Clementine mandarin fruit at ambient temperature. Journal of Food Agriculture Environment, 3, 34-36.

Magazin, N., Gvozdenovi, D., Keserovi, Z. and Milic, B. (2010) Fruit quality of Granny Smith apples picked at different harvest times and treated with 1-MCP. Fruits, 65, 191-197.

McGuire, R.G. (1992) Reporting of objective colour measurements. HortScience, 27 (12), 1254-1255.

Meyer, M.D. and Terry, L.A. (2010) Fatty acid and sugar composition of avocado, cv. Hass, in response to treatment with an ethylene scavenger or 1-methylcyclopropene to extend storage life. Food Chemistry, 121, 1203-1210.

Mitra, S.K. and Baldwin, E.Z. (1997) Mango. In: "Postharvest Physiology and Storage of Tropical and Tropical Fruits", (Mitra S.K., ed.), pp 85-122. CAB International, West Bengal, India.

M-STAT, (1993) A microcomputer program for the design, arrangement and analysis of agronomic research experiments. Michigan State University.

Narayana, C.K., Pal, R.K. and Roy, S.K. (1996) Effect of pre-storage treatments and temperature regimes on shelf life and respiratory behaviour of ripe Baneshan mango. Journal of Food Science and Technology, 33, 79-82.

Nunes, C.N., Emond, J.P., Bredcht, J.K., Dea, S. and Prooulx, E. (2007) Quality curves for mango fruit stored at chilling and non chilling temperatures. Journal of Food Quality, 30, 104-120.

Özkaya, O., Yildirimb, D., Dündara, Ö. and Seyhan Tükel, S. (2016) Effects of 1methylcyclopropene (1-MCP) and modified atmosphere packaging on postharvest storage quality of nectarine fruit. Scientia Horticulturae, 198, 454-461.

Egypt. J. Hort. Vol. 43, No.2 (2016) 
Pietro, R., Cocci, E., Romani, S., Sacchetti, G. and Rosa, M. D. (2009) Effect of 1MCP treatment and $\mathrm{N}_{2} \mathrm{O}$ MAP on physiological and quality changes of fresh cut pineapple. Postharvest Biology and Technology, 51 (3), 371-377.

Pongprasert, N. and Srilaong, V. (2014) A novel technique using 1-MCP microbubbles for delaying postharvest ripening of banana fruit. Postharvest Biology and Technology, 95, 42-45.

Porat, R., Weiss, B., Cohen, L., Daus, A., Goren, R. and Droby, S. (1999) Effects of ethylene and 1-methylcyclopropene on the postharvest qualities of 'Shamouti' oranges. Postharvest Biology and Technology, 15 (2), 155-163.

Prusky, D. and Keen, N.T. (1993) Involvement of preformed antifungal compounds and the resistance of subtropical fruits to fungal decay. Plant Disease, 77, 114-119.

Rathore, H. A., Masud, T., Sammi, S. and Soomro, H. A. (2007) Effect of storage on physico-chemical composition and sensory properties of mango (Mangifera indica L.) variety Dosehari. Pakistan Journal of Nutrition, 6 (2), 143-148.

Rizzolo, A., Grassi, M. and Vanoli, M. (2014) 1-Methylcyclopropene application, storage temperature and atmosphere modulate sensory quality changes in shelf-life of 'Abbé Fétel' pears. Postharvest Biology and Technology, 92, 87-97

Saltveit, M.E. (1999) Effect of ethylene on quality of fresh fruits and vegetables. Postharvest Biology and Technology, 15, 279-292.

Singh, V.K. and P. Neelam. (2008) Response of 1-methylcyclopropene on activities of pectin hydrolases and associated post-harvest physiological changes in different mango cultivars. Journal of Horticulture, 65, 20-24.

Sisler, E. C. and Serek, M. (1997) Inhibitors of ethylene responses in plants at the receptor level: Recent developments. Physiologia Plantarum, 100, 577-582.

Sivakumar, D., Van Deventer, F., Terry, L.A., Polenta, G.A. and Korsten, L. (2012) Combination of 1-methylcyclopropene treatment and controlled atmosphere storage retains overall fruit quality and bioactive compounds in mango. Journal of Science Food and Agriculture, 92, 821-830.

Smirnoff, N. (1996) The function and metabolism of ascorbic acid in plants. Annals of Botany, 78, 661-669.

Steel, R.G.D., Torrie, J.H. and Diskey, D.A. (1997) "Principles and Procedures of Statistics. A Biometrical Approach", $3^{\text {rd }}$ ed., McGrow-Hill Publishing Company, New York, USA, 336-376.

Sudha, R., Amutha, R., Muthulaksmi, S., Baby Rani, W., Indira, K. and Mareeswari, P. (2007) Influence of pre and postharvest chemical treatments on physical characteristics of sapota (Achras sapota L.) Var. PKM 1. Research Journal of Agriculture and Biological Science, 3 (5), 450-452. 
Tavares, S., Castro, P.R.C., Kluge, R.A. and Jacomino, A.P. (2004) Growth postharvest regulators in Tahiti acid lime. Laranja, 25 (1), 209-224.

Vanoli, M., Rizzolo, A. and Grassi, M. (2013) Fruit quality and sensory characteristics of 1-MCP treated 'Abbé Fètel' pears after storage under dynamic controlled atmosphere at different temperatures. In: International Congress CAMA 2013. XI International Controlled and Modified Atmosphere Research Conference, 3-7 June, Trani, Italy.

Voss, D.H. (1992) Relating colourimeter measurement of plant colour to the royal horticultural society colour chart. HortScience, 27 (12), 1256-1260.

Wahdan, M.T., Habib, S.E., Bassal, M.A. and Qaoud, E. M. (2011) Effect of calcium chloride and gibberellic acid on storability of 'Succary Abiad' mango fruits under cold storage. Journal of American Science, 7 (5), 493-501

Wang, B., Wang, J., Feng, X., Lin, L., Zhao, Y. and Jiang, W. (2009) Effects of 1MCP and exogenous ethylene on fruit ripening and antioxidants in stored mango. Plant Growth Regulators, 57, 185-192.

Wang, K.L., Li, H. and Ecker, J.R. (2002) Ethylene biosynthesis and signaling networks. Plant Cell, 14, 131-151.

Watkins, C. and Harman, J. (1981) Use of penetrometer to measure flesh firmness of fruit. Orchadist, N. Z., 14-16.

Watkins, C.B. (2006) The use of 1-methylcyclopropene (1-MCP) on fruits and vegetables. Biotechnology Advances, 24, 389-409.

Watkins, C.B. (2008) Overview of 1-methylcyclopropene trials and uses for edible horticultural crops. HortScience, $\mathbf{4 3}$ (1), 86-94.

Wills, R., Mc Glasson, B., Graham, D. and Joyce, D. (1998) Postharvest: an introduction to the physiology and handling of fruit, vegetables and ornamentals. UNSW Press, Sydney, Australia, 262 p.

Win, T. O., Srilaong, V., Heyes, J., Kyu, K. L. and Kanlayanarat, S. (2006) Effects of different concentrations of 1-MCP on the yellowing of West Indian lime (Citrus aurantifolia, Swingle) fruit. Postharvest Biology and Technology, 42, 23-30.

Wongkhot, A., Rattanapanone, N. and Chanasut, U. (2012) Brim A, total acidity and total soluble solids correlate to total carotenoid content as indicators of the ripening process of six Thai mango fruit cultivars. Chiang Mai University Journal of Natural Sciences, 11 (1), 97-103.

Zumreoglu-Karan, B. (2006) The coordination chemistry of vitamin C: An overview. Coordination Chemistry Reviews, 250, 2295-2307. 


\section{زيادة القدرة التخزينية لثمار المانجو صنف زبلده بالمعاملة}

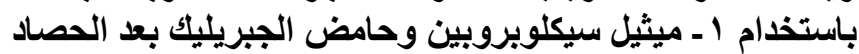

محمود علي أحمد محمد* أحد فتحي عبد الخالق*** وعلال محمد ربيع عبد اللطيف

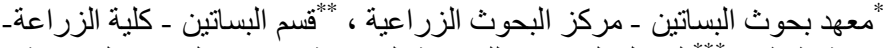

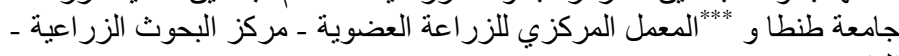

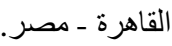

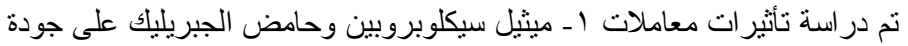

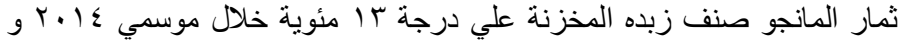

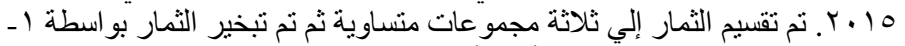

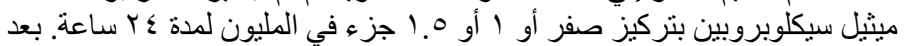

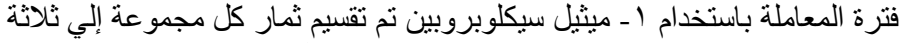

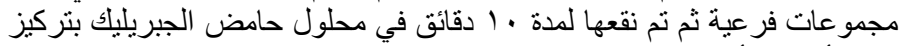

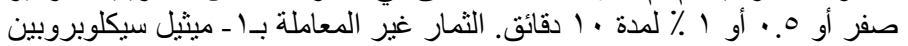

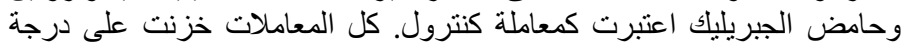

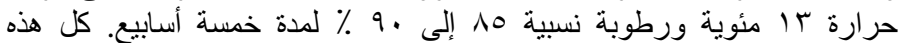

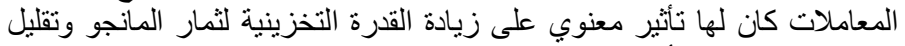

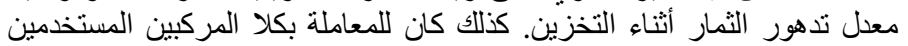

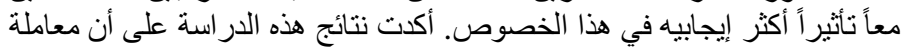

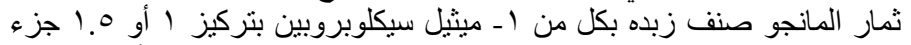

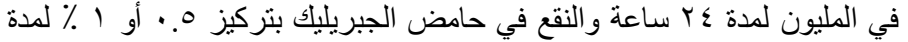

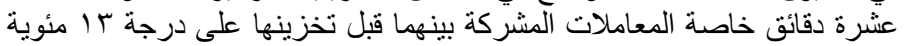

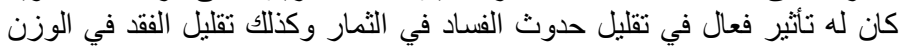

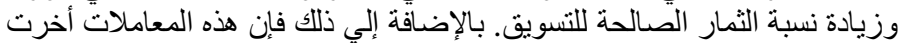

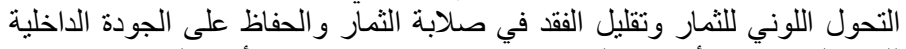

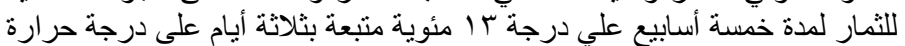
الغرفة كفترة عمر تسويقي. 